\title{
A quasinormal criterion of meromorphic functions and its application
}

Pai Yang ${ }^{*}$

\section{"Correspondence:}

yangpai@cuit.edu.cn

Department of Mathematics,

Nanjing University, Nanjing, 210093,

P.R. China

College of Applied Mathematics,

Chengdu University of Information

Technology, Chengdu, 610225,

P.R. China

\begin{abstract}
In this paper, we obtain a quasinormal criterion of meromorphic functions and give an example of an application in the value distribution theory. More than anything, we provide a general method to solve some problems in the value distribution theory.
\end{abstract}

MSC: 30D35; 30D45

Keywords: elliptic function; quasinormal criterion; characteristic function

\section{Introduction}

We use the following notation. Let $\mathbb{N}$ denote the set of positive integers. Let $\mathbb{C}$ be complex plane and $D$ be a domain in $\mathbb{C}$. For $z_{0} \in \mathbb{C}$ and $r>0, \Delta\left(z_{0}, r\right)=\left\{z|| z-z_{0} \mid<r\right\}, \Delta^{\prime}\left(z_{0}, r\right)=$ $\left\{z|0<| z-z_{0} \mid<r\right\}$ and $\Delta=\Delta(0,1)$. Let $n(D, f)$ denote the number of poles of $f(z)$ in $D$ (counting multiplicities) and $\bar{n}(D, f)$ denote the number of poles of $f(z)$ in $D$ (not counting multiplicities). We write $f_{n} \stackrel{x}{\Rightarrow} f$ in $D$ to indicate that the sequence $\left\{f_{n}\right\}$ converges to $f$ in the spherical metric uniformly on compact subsets of $D$ and $f_{n} \Rightarrow f$ in $D$ if the convergence is in the Euclidean metric. For $f$ meromorphic in $D$, set

$$
f^{\#}(z):=\frac{\left|f^{\prime}(z)\right|}{1+|f(z)|^{2}} \quad \text { and } \quad S(D, f):=\frac{1}{\pi} \iint_{D}\left[f^{\#}(z)\right]^{2} \mathrm{~d} x \mathrm{~d} y
$$

The Ahlfors-Shimizu characteristic is defined by $T_{0}(r, f)=\int_{0}^{r} \frac{S(t, f)}{t} \mathrm{~d} t$. Let $T(r, f)$ denote the usual Nevanlinna characteristic function. Since $T(r, f)-T_{0}(r, f)$ is bounded as a function of $r$, we can replace $T_{0}(r, f)$ with $T(r, f)$ in this paper.

Recall that an elliptic function [1] is a meromorphic function $h$ defined in $\mathbb{C}$ for which there exist two nonzero complex numbers $\omega_{1}$ and $\omega_{2}$ with $\omega_{1} / \omega_{2}$ not real such that $h(z+$ $\left.\omega_{1}\right)=h\left(z+\omega_{2}\right)=h(z)$ for all $z$ in $\mathbb{C}$.

Recall that a family $\mathcal{F}$ of functions meromorphic in $D$ is said to be quasinormal in $D$ [2] if from each sequence $\left\{f_{n}\right\} \subset \mathcal{F}$ one can extract a subsequence $\left\{f_{n_{k}}\right\}$ which converges locally uniformly with respect to the spherical metric in $D \backslash E$, where the set $E$ (which may depend on $\left.\left\{f_{n_{k}}\right\}\right)$ has no accumulation points in $D$. If $E$ can always be chosen to satisfy $|E| \leq v, \mathcal{F}$ is said to quasinormal of order $v$ in $D$. Thus a family is quasinormal of order 0 in $D$ if and only if it is normal in $D$. The family $\mathcal{F}$ is said to be (quasi)normal at $z_{0} \in D$ if it is (quasi)normal in some neighborhood of $z_{0}$. Thus $\mathcal{F}$ is quasinormal in $D$ if and only if it is quasinormal at each point $z \in D$. On the other hand, $\mathcal{F}$ fails to be quasinormal of order

○2014 Yang; licensee Springer. This is an Open Access article distributed under the terms of the Creative Commons Attribution License (http://creativecommons.org/licenses/by/2.0), which permits unrestricted use, distribution, and reproduction in any medium, provided the original work is properly cited. 
$v$ in $D$ precisely when there exist points $z_{1}, z_{2}, \ldots, z_{v+1}$ in $D$ and a sequence $\left\{f_{n}\right\} \subset \mathcal{F}$ such that no subsequence of $\left\{f_{n}\right\}$ is normal at $z_{j}(j=1,2, \ldots, v+1)$.

In 2007, Nevo et al. proved the following quasinormal criterion.

Theorem A [3, Theorem 1] Let $\mathcal{F}$ be a family of meromorphic functions in $D$, all of whose zeros have multiplicity at least $k+1$. If there exists a holomorphic function $H$ univalent in $D$ such that $f^{(k)}(z) \neq H^{\prime}(z)$ for all $f \in \mathcal{F}$ and all $z \in D$, then $\mathcal{F}$ is quasinormal of order 1 in $D$.

For $k \geq 2$, we extend Theorem A in this paper. In fact, we obtain the following result.

Theorem 1.1 Let $k \geq 2$ be an integer, and let $\mathcal{F}$ be a family of meromorphic functions in $D$, all of whose zeros have multiplicity at least $k+1$. Let $H$ be a nonconstant meromorphic function. Suppose that there exists $v \in \mathbb{N}$ such that for each $a^{*} \in \mathbb{C}, \bar{n}\left(D, \frac{1}{H(z)-a^{*}}\right) \leq v$. If $f^{(k)}(z) \neq H^{\prime}(z)$ for all $f \in \mathcal{F}$ and all $z \in D$, then $\mathcal{F}$ is quasinormal of order $v$ in $D$.

Remark 1.1 For $k \geq 2$, Theorem A is the special case of Theorem 1.1 with $v=1$ and $H^{\prime}(z) \neq$ $0, \infty$ for all $z \in D$. Unfortunately, the restricted condition that $H^{\prime}(z) \neq 0, \infty$ largely restricts the applications of Theorem A, so it is important to remove the condition's restriction.

For convenience, we give a generalized form of Theorem 1.1.

Proposition 1.2 Let $k \geq 2$ be an integer, and let $\left\{f_{n}\right\}$ be a family of meromorphic functions in $D$, all of whose zeros have multiplicity at least $k+1$. Let $H$ be a nonconstant meromorphic function, and there exists $v \in \mathbb{N}$ such that for each $a^{*} \in \mathbb{C}, \bar{n}\left(D, \frac{1}{H(z)-a^{*}}\right) \leq v$. Let $\left\{h_{n}\right\}$ be a family of meromorphic functions in $D$ such that $h_{n}$ and $H^{\prime}$ have the same zeros and poles with the same multiplicity, and $h_{n}(z) \stackrel{\chi}{\Rightarrow} H^{\prime}(z)$ in $D$. If $f_{n}^{(k)}(z) \neq h_{n}(z)$ for all $n \in \mathbb{N}$ and all $z \in D$, then $\left\{f_{n}\right\}$ is quasinormal of order $v$ in $D$.

Moreover, for each subsequence $\left\{f_{n_{k}}\right\}$ of $\left\{f_{n}\right\}$, there exist a subsequence of $\left\{f_{n_{k}}\right\}$ (still denoted by $\left.\left\{f_{n_{k}}\right\}\right)$ and a corresponding point set $E$ which has no accumulation points in D such that:

(1) $f_{n_{k}}(z) \stackrel{\chi}{\Rightarrow} f(z)$ in $D \backslash E$, where $f(z)$ is meromorphic or identically infinite there;

(2) for each $\tilde{a} \in E, H(\tilde{a}) \neq \infty$ and no subsequence of $\left\{f_{n_{k}}\right\}$ is normal at $\tilde{a}$;

(3) for each $\tilde{a} \in E$, there exist $r_{\tilde{a}}>0$ and $N_{\tilde{a}}>0$ such that for sufficiently large $k$, $n\left(\Delta\left(\tilde{a}, r_{\tilde{a}}\right), \frac{1}{f_{n_{k}}}\right)<N_{\tilde{a}}$, where $r_{\tilde{a}}$ and $N_{\tilde{a}}$ only depend on $\tilde{a}$; and

(4) for each $\tilde{a} \in E, f(z)=\int_{\tilde{a}}^{z} \int_{\tilde{a}}^{\zeta_{1}} \cdots \int_{\tilde{a}}^{\zeta_{k-1}} H^{\prime}\left(\zeta_{k}\right) \mathrm{d} \zeta_{k} \mathrm{~d} \zeta_{k-1} \cdots \mathrm{d} \zeta_{1}$ in $D \backslash E$.

The value distribution theory of meromorphic functions occupies one of the central places in complex analysis which now has been applied to complex dynamics, complex differential and functional equations, Diophantine equations, and others.

In his excellent paper [4], Hayman studied the value distribution of certain meromorphic functions and their derivatives under various conditions. Among other important results, he proved that if $f(z)$ is a transcendental meromorphic function in the plane, then either $f(z)$ assumes every finite value infinitely often, or every derivative of $f(z)$ assumes every finite nonzero value infinitely often. This result is known as Hayman's alternative. Thereafter, the value distribution of derivatives of transcendental functions continued to be studied.

In 1995, Bergweiler and Eremenko proved the following result. 
Theorem B [5, Theorem 3] Let $f$ be a meromorphic function of finite order in $\mathbb{C}$. Iff has infinitely many multiple zeros, then $f^{\prime}$ assumes every finite nonzero value infinitely often.

In 2008, Pang et al. obtained the following result.

Theorem C [6, Theorem 1] Let $f$ be a transcendental meromorphic function in $\mathbb{C}$, all but finitely many of whose zeros are multiple, and let $R(\equiv 0)$ be a rational function. Then $f^{\prime}-R$ has infinitely many zeros.

$R$ is a small function compared with $f$ in Theorem C. Specifically, $T(r, R)=o\{T(r, f)\}$ as $r \rightarrow \infty$ in Theorem C. A natural problem arises: What can we say if the rational function $R$ in Theorem $\mathrm{C}$ is replaced by a more general small function $\alpha(z)$ ? In this direction, we obtain the following result.

Theorem 1.3 Let $k \geq 2$ bean integer, let $f(z)$ be a meromorphic function in $\mathbb{C}$, and let $\alpha(z)=$ $R(z) h(z)(\neq \equiv)$, where $h(z)$ is an elliptic function and $R(z)$ is a rational function. Suppose that all but finitely many zeros of $f$ have multiplicity at least $k+1$ and $T(r, \alpha)=o\{T(r, f)\}$ as $r \rightarrow \infty$. Then the equation $f^{(k)}(z)=\alpha(z)$ has infinitely many solutions (including the possibility of infinitely many common poles of $f(z)$ and $\alpha(z))$.

\section{Preliminary lemmas}

Lemma 2.1 [7, Corollary 2] If h $(z)$ is a nonconstant elliptic function with primitive periods $\omega_{1}, \omega_{2}$, where $\omega_{1} / \omega_{2}$ is not real, then $T(r, h)=A r^{2}(1+o(1))$ as $r \rightarrow \infty$, where $A>0$ is a constant.

Lemma 2.2 Let $\mathcal{F}$ be a family of functions meromorphic in $D$, all of whose zeros have multiplicity at least $k$, and suppose that there exists $A \geq 1$ such that $\left|f^{(k)}(z)\right| \leq A$ whenever $f(z)=0$. Then if $\mathcal{F}$ is not normal at $z_{0}$, there exist, for each $0 \leq \alpha \leq k$,

(a) points $z_{n}, z_{n} \rightarrow z_{0}$;

(b) functions $f_{n} \in \mathcal{F}$; and

(c) positive numbers $\rho_{n} \rightarrow 0$

such that $\rho_{n}^{-\alpha} f_{n}\left(z_{n}+\rho_{n} \zeta\right)=g_{n}(\zeta) \stackrel{\chi}{\Rightarrow} g(\zeta)$ in $\mathbb{C}$, where $g$ is a nonconstant meromorphic function in $\mathbb{C}$ such that $g^{\#}(\zeta) \leq g^{\#}(0)=k A+1$. In particular, $g$ has order at most 2 .

This is the local version of [8, Lemma 2] (cf. [9, Lemma 1]; [10, pp.216-217]). The proof consists of a simple change of variable in the result cited from [8]; $c f$. [11, pp.299-300].

Lemma 2.3 [12, Lemma 3.6] Let $\left\{f_{n}\right\}$ be a family of functions meromorphic in $\Delta\left(z_{0}, r\right)$. Suppose that $f_{n} \stackrel{\chi}{\Rightarrow} f$ in $\Delta^{\prime}\left(z_{0}, r\right)$, where $f$ is a nonconstant meromorphic function or $f \equiv \infty$ in $\Delta^{\prime}\left(z_{0}, r\right)$. If there exists $M_{0}>0$ such that for each $n, n\left(\Delta\left(z_{0}, r\right), \frac{1}{f_{n}}\right)<M_{0}$, then there exists $M_{1}>0$ such that $S\left(\Delta\left(z_{0}, r / 2\right), f_{n}\right)<M_{1}$.

Lemma 2.4 [3, Lemma 3] Let $k$ be a positive integer, let $\left\{f_{n}\right\}$ be a family of meromorphic functions in $D$, and let $\left\{\psi_{n}\right\}$ be a family of holomorphic functions in $D$ such that $\psi_{n} \Rightarrow \psi$, where $\psi(z) \neq 0, \infty$ in $D$. If for all $n \in \mathbb{N}$ and all $z \in D, f_{n}(z) \neq 0$ and $f_{n}^{(k)}(z) \neq \psi_{n}(z)$, then $\left\{f_{n}\right\}$ is normal in $D$. 
Lemma 2.5 Let $\left\{a_{j}\right\}$ be a sequence in $D$ which has no accumulation points in $D$, and let $\left\{\psi_{n}\right\}$ be a family of holomorphic functions in D such that $\psi_{n} \Rightarrow \psi$ in $D$, where $\psi \neq 0, \infty$ in $D$. Let $\left\{f_{n}\right\}$ be a family of meromorphic functions in $D$, all of whose zeros have multiplicity at least $k+1$, such that $f_{n}^{(k)}(z) \neq \psi_{n}(z)$ for all $n \in \mathbb{N}$ and all $z \in D$. Suppose that:

(a) no subsequence of $\left\{f_{n}\right\}$ is normal at $a_{1}$;

(b) $f_{n}(z) \stackrel{x}{\Rightarrow} f(z)$ in $D \backslash\left\{a_{j}\right\}_{j=1}^{\infty}$.

Then

(c) there exists $r_{1}>0$ such that $f_{n}$ has a single (multiple) zero in $\Delta\left(a_{1}, r_{1}\right)$ for sufficiently large $n$;

(d) there exists $r_{2}>0$ such that for each $0<r<r_{2}, f_{n}$ has a single simple pole in $\Delta\left(a_{1}, r\right)$ for sufficiently large $n$; and

(e) $f(z)=\int_{a_{1}}^{z} \int_{a_{1}}^{\zeta_{1}} \cdots \int_{a_{1}}^{\zeta k-1} \psi\left(\zeta_{k}\right) \mathrm{d} \zeta_{k} \mathrm{~d} \zeta_{k-1} \cdots \mathrm{d} \zeta_{1}$ for $z \in D \backslash\left\{a_{j}\right\}_{j=2}^{\infty}$. Equivalently, $f$ extends to an analytic function in $D \backslash\left\{a_{j}\right\}_{j=2}^{\infty}$ such that $f^{(k)}(z)=\psi(z)$ and $f^{(j)}\left(a_{1}\right)=0$ for $j=0,1,2, \ldots, k-1$.

Remark 2.1 Since Lemma 2.5 is not stated explicitly in [3], let us indicate how it follows from the results of that paper. Suppose first that (c) has been shown to hold. By Lemma 7 in [3], (d) and (e) hold. Next, suppose that (c) do not hold. Similar to the treatment in Case 2 (pp.13-16) of the proof of Theorem 1 in [3], we can finally derive a contradiction.

Lemma 2.6 Let $k$ be a positive integer, and let $\left\{f_{n}\right\}$ be a family of meromorphic functions in $D$, all of whose zeros have multiplicity at least $k+1$. Let $\left\{h_{n}\right\}$ be a family of meromorphic functions in $D$ such that $h_{n}(z) \stackrel{x}{\Rightarrow} H^{\prime}(z)$ in $D$, where $H$ is a holomorphic univalent function in $D$. If $f_{n}^{(k)}(z) \neq h_{n}(z)$ for all $n \in \mathbb{N}$ and all $z \in D$, then $\left\{f_{n}\right\}$ is quasinormal of order 1 in $D$.

Lemma 2.6 can be proved by an exactly analogous argument as in the proof of Theorem 1 in [3]. In fact, there is no essential difference between Lemma 2.6 and Theorem 1 in [3], so we do not give the proof of Lemma 2.6.

Lemma 2.7 [13, Lemma 12] Let $R$ be a nonconstant rational function satisfying $R^{\prime}(z) \neq 0$ in $\mathbb{C}$. Then either $R(z)=a z+b$ or $R(z)=\frac{a}{(z+c)^{n}}+b$, where $n \in \mathbb{N}$ and $a(\neq 0), b, c \in \mathbb{C}$.

Lemma 2.8 [14, Theorem 1] Let $k$ be a positive integer, let $f$ be a transcendental meromorphic function in $\mathbb{C}$, and let $R(\equiv 0)$ be a rational function. If all but finitely many zeros off have multiplicity at least $k+1$, then $f^{(k)}-R$ has infinitely many zeros.

Remark 2.2 The proof of Lemma 2.8 is based, quite naturally, on a combination of ideas from [15] and [6]. In fact, fully understanding the ideas and methods in [15] and [6], one can give the proof of Lemma 2.8 without difficulty.

Lemma 2.9 [16, Lemma 6] Let $k$ and $l$ be positive integers, and let $R(z)$ be a rational function, all of whose zeros have multiplicity at least $k$. If $R^{(k)}(z) \neq z^{-l}$ in $\mathbb{C}$, then $R(z)$ is a constant.

Lemma 2.10 [16, Lemma 10] Let $k, l$ be positive integers with $l \geq k+1$, let $\left\{\varphi_{n}\right\}$ be a family of holomorphic functions, and let $\left\{f_{n}\right\}$ be a family of meromorphic functions, all of whose poles are multiple and all of whose zeros have multiplicity at least $k+1$. Suppose that: 
(1) $f_{n}$ and $\varphi_{n}$ are defined in $\Delta_{R_{n}}$, where the positive sequence $R_{n}$ increases to $\infty$;

(2) $\varphi_{n} \Rightarrow 1$ in $\mathbb{C}$;

(3) $f_{n}^{(k)}(z) \neq \varphi_{n}(z) / z^{l}$ for $z \in \Delta_{R_{n}}$;

(4) $f_{n} \stackrel{x}{\Rightarrow} f$ in $\mathbb{C}$; and

(5) $f(1)=0$.

Then $f$ has a zero in $\Delta$.

Lemma 2.11 Let $f$ be a nonconstant meromorphic function of finite order in $\mathbb{C}$, all of whose zeros have multiplicity at least $k+1$. If $f^{(k)}(z) \neq 1$ in $\mathbb{C}$, then

$$
f(z)=\frac{1}{k !} \frac{(z-a)^{k+1}}{z-b}
$$

for some $a$ and $b(\neq a)$ in $\mathbb{C}$.

This follows from results in [17], specifically Lemma 6 (whose proof depends in an essential fashion on Corollary 3 of [5]) and Lemma 8. As an immediate consequence of Lemma 2.11, we have the following result, which appears as Lemma 9 of [17].

Lemma 2.12 [17, Lemma 9] Let $k$ be a positive integer, and let $f$ be a meromorphicfunction of finite order in $\mathbb{C}$, all of whose poles are multiple and whose zeros all have multiplicity at least $k+1$. If $f^{(k)}(z) \neq c$ for some constant $c \neq 0$ and all $z \in \mathbb{C}$, then $f(z)$ is a constant.

\section{Auxiliary lemmas}

Lemma 3.1 Let $\left\{f_{n}\right\}$ and $\left\{\psi_{n}\right\}$ be families of meromorphic functions in $D$, and let $f(z)$ and $\psi(z)$ be meromorphic functions in D. Suppose that:

(a) $f_{n}(z) \stackrel{\chi}{\Rightarrow} f(z)$ and $\psi_{n}(z) \stackrel{x}{\Rightarrow} \psi(z)$ in $D$, and

(b) $f_{n}^{(k)}(z) \neq \psi_{n}(z)$ in $D$.

Then, either $f^{(k)}(z) \equiv \psi(z)$ orf $f^{(k)}(z) \neq \psi(z)$ in $D$.

Proof Suppose that $f^{(k)}(z) \not \equiv \psi(z)$ in $D$. Set $A:=f^{-1}(\infty) \cup \psi^{-1}(\infty) \cup\left(f^{(k)}-\psi\right)^{-1}(0)$. By (a) and (b), we have

$$
\infty \neq \frac{1}{f_{n}^{(k)}-\psi_{n}} \Rightarrow \frac{1}{f^{(k)}-\psi} \quad \text { in } D \backslash A .
$$

Since $\frac{1}{f_{n}^{(k)}-\psi_{n}}$ is holomorphic in $D$ and $A$ has no accumulation points in $D$, we have

$$
\frac{1}{f_{n}^{(k)}-\psi_{n}} \Rightarrow \frac{1}{f^{(k)}-\psi} \quad \text { in } D
$$

Thus $\frac{1}{f^{(k)}-\psi}$ is a holomorphic function in $D$ and then $f^{(k)}-\psi \neq 0$ in $D$.

In order to show that $f^{(k)} \neq \psi$ in $D$, we need only show that $f$ and $\psi$ have no common poles in $D$. Otherwise, we assume that $z_{0} \in D$ is a pole of order $m_{1}$ of $f$ and a pole of order $m_{2}$ of $\psi$. Set $m:=\max \left\{m_{1}+k, m_{2}\right\}$. Obviously, $z_{0}$ is a zero of $\frac{1}{f^{(k)}-\psi}$ of order at most $m$.

By (a) and Hurwitz' theorem, there exists $\delta^{*}$ such that $\Delta\left(z_{0}, 2 \delta^{*}\right) \subset D$ and for each $\delta \in$ $\left(0, \delta^{*}\right), f_{n}$ and $b_{n}$ have at least $m_{1}$ and $m_{2}$ (counting multiplicities) poles respectively in $\Delta\left(z_{0}, \delta\right)$ for sufficiently large $n$. By (b), $f_{n}$ and $\psi_{n}$ have no common poles in $\Delta\left(z_{0}, \delta\right)$, and 
hence $f_{n}^{(k)}-\psi_{n}$ has at least $m_{1}+k+m_{2}$ poles (counting multiplicities) in $\Delta\left(z_{0}, \delta\right)$. Since $\delta$ can be made arbitrarily small, $z_{0}$ is a zero of $\frac{1}{f^{(k)}-\psi}$ of order at least $m_{1}+k+m_{2}$ by (3.1). Thus $m_{1}+k+m_{2}>m$. This is a contradiction.

Lemma 3.2 Let $k$ be a positive integer, and let $\left\{f_{n}\right\}$ be a family of meromorphic functions in $\Delta$, all of whose zeros have multiplicity at least $k+1$. Let $\left\{b_{n}\right\}$ be a sequence of meromorphic functions in $\Delta$ such that $b_{n}(z) \stackrel{x}{\Rightarrow} b(z)$ in $\Delta$, where $b(\not \equiv 0)$ is a meromorphic function and $b(0)=0$. Suppose that:

(a) $b$ and $b_{n}$ have the same zeros and poles with the same multiplicity;

(b) for all $n \in \mathbb{N}$ and all $z \in D, f_{n}^{(k)}(z) \neq b_{n}(z)$;

(c) there exists points $z_{n}$ in $\Delta$ such that $f_{n}\left(z_{n}\right)=0$ and $z_{n} \rightarrow 0$; and

(d) $f_{n}(z) \stackrel{\chi}{\Rightarrow} f(z)$ in $\Delta^{\prime}$, where $f(z)$ is a meromorphic function in $\Delta^{\prime}$.

Then $f^{(k)}(z) \equiv b(z)$ in $\Delta^{\prime}$.

Proof Set $F_{n}(z):=\frac{f_{n}^{(k)}(z)}{b_{n}(z)}$. By (a) and (b), $f_{n}^{(k)}(0) \neq b_{n}(0)=0$, and hence $F_{n}(0)=\infty$. Since all zeros of $\left\{f_{n}(z)\right\}$ have multiplicity at least $k+1$, we have $f_{n}(0) \neq 0$. Hence $z_{n} \neq 0$ and $F_{n}\left(z_{n}\right)=0$ for sufficiently large $n$. Since $F_{n}(0)=\infty$ and $F_{n}\left(z_{n}\right)=0$ for sufficiently large $n,\left\{F_{n}(\zeta)\right\}$ is not equicontinuous at 0 , and hence $\left\{\frac{f_{n}^{(k)}(z)}{b_{n}(z)}-1\right\}$ is not normal at 0 .

By Lemma 3.1, we have either $f^{(k)}(z) \equiv b(z)$ or $f^{(k)}(z) \neq b(z)$ in $\Delta^{\prime}$. Suppose that $f^{(k)}(z) \neq$ $b(z)$ in $\Delta^{\prime}$. By the assumptions, there exists $\delta>0$ such that $f(z)$ has no poles on $\Gamma(0, \delta)$ and $b(z)$ has no zeros on $\Gamma(0, \delta)$. Thus, we have

$$
\infty \neq \frac{1}{\frac{f_{n}^{(k)}(z)}{b_{n}(z)}-1} \Rightarrow \frac{1}{\frac{f^{(k)}(z)}{b(z)}-1}, \quad z \in \Gamma(0, \delta) .
$$

By the maximum principle, (3.2) holds in $\Delta(0, \delta)$, and then $\left\{\frac{f_{\frac{f}{(k)}(z)}(z)}{b_{n}(z)}\right.$ is normal at 0 . This is a contradiction. Thus $f^{(k)}(z) \equiv b(z)$ in $\Delta^{\prime}$.

Lemma 3.3 Let $k$ be a positive integer, let $\left\{f_{n}\right\}$ be a family of meromorphic functions in $D$, and let $\left\{h_{n}\right\}$ be a family of meromorphic functions in $D$ such that $h_{n} \stackrel{x}{\Rightarrow} h$ in $D$, where $h \neq 0, \infty$. If all $n \in \mathbb{N}$ and all $z \in D, f_{n}(z) \neq 0$ and $f_{n}^{(k)}(z) \neq h_{n}(z)$, then $\left\{f_{n}\right\}$ is normal in $D$.

Proof By Lemma 2.4, it suffices to prove that $\left\{f_{n}\right\}$ is normal at points which $h$ has poles or zeros. Without loss of generality, we assume that $D=\Delta, h(z)=z^{l} b(z)$, where $b(z) \neq 0, \infty$ in $\Delta$ and $l(\neq 0)$ is an integer. Then $\left\{f_{n}\right\}$ is normal in $\Delta^{\prime}$.

Suppose that $\left\{f_{n}\right\}$ is not normal at 0 . Taking a subsequence and renumbering, we may assume that no subsequence of $\left\{f_{n}\right\}$ is normal at 0 . Since $f_{n}(z) \neq 0$ in $\Delta$, there exists $r>0$ such that $\Delta_{2 r} \subset \Delta$ and $f_{n} \Rightarrow 0$ in $\Delta_{2 r}^{\prime}$. By the argument principle, we have, for sufficiently large $n$,

$$
n\left(r, \frac{1}{f_{n}^{(k)}-h_{n}}\right)-n\left(r, f_{n}^{(k)}-h_{n}\right)=\frac{1}{2 \pi i} \int_{|z|=r} \frac{f_{n}^{(k+1)}-h_{n}^{\prime}}{f_{n}^{(k)}-h_{n}} \mathrm{~d} z=\frac{1}{2 \pi i} \int_{|z|=r} \frac{h^{\prime}}{h} \mathrm{~d} z=l .
$$

Since $f_{n}^{(k)}(z) \neq h_{n}(z)$, we have $-n\left(r, f_{n}^{(k)}-h_{n}\right)=l$. Thus $l<0$ and $f_{n}$ has poles (otherwise $f_{n} \stackrel{x}{\Rightarrow} \infty$ in $\left.\Delta^{\prime}\right)$ which are different from the poles of $h_{n}$. Hence $n\left(r, f_{n}^{(k)}-h_{n}\right)>-l$. This is a contradiction. 
Lemma 3.4 Let $k$ be a positive integer, and let $\left\{f_{n}\right\}$ be a family of meromorphic functions in $D$, all of whose zeros have multiplicity at least $k+1$. Let $\left\{\psi_{n}\right\}$ be a family of meromorphic functions in $D$ such that $\psi_{n} \stackrel{\chi}{\Rightarrow} \psi$, where $\psi(z) \not \equiv 0, \infty$ in $D$. If $f_{n}^{(k)} \neq \psi_{n}$ for all $n \in \mathbb{N}$ and all $z \in D$, then $\left\{f_{n}\right\}$ is quasinormal in $D$.

Proof It suffices to show that $\left\{f_{n}\right\}$ is quasinormal in a neighborhood of each point of $D$. Let $p \in D$. There exists $t>0$ such that $\Delta(p, t) \subset D$ and $\psi$ is holomorphic and does not vanish in $\Delta^{\prime}(p, t)$. By Lemma 2.6, $\left\{f_{n}\right\}$ is quasinormal in $\Delta^{\prime}(p, t)$.

Suppose now that $\left\{f_{n}\right\}$ is not quasinormal at $p$. Then there exist points $z_{j} \in \Delta^{\prime}(p, \delta)$ $(j=1,2, \ldots)$ and a subsequence of $\left\{f_{n}\right\}$ (still denoted by $\left.\left\{f_{n}\right\}\right)$ such that $z_{j} \rightarrow p$ and no subsequence of $\left\{f_{n}\right\}$ is normal at any $z_{j}, j=1,2, \ldots$. Set $E:=\left\{z_{j}: j=1,2, \ldots\right\}$. Taking a subsequence of $\left\{f_{n}\right\}$ (still denoted by $\left\{f_{n}\right\}$ ), we may assume that $f_{n} \stackrel{\chi}{\Rightarrow} H$ in $\Delta^{\prime}(p, \delta) \backslash E$. By Lemma 2.5 , we have $H^{(k)} \equiv \psi$ and $H\left(z_{j}\right)=0$. It follows that $H$ is holomorphic in $\Delta^{\prime}(p, t)$ and $H^{(k)} \equiv \psi$ there. Moreover, since $\psi$ has no essential singularity at $p$, the same is true of $H$. But that $H\left(z_{j}\right)=0$ for $j=1,2, \ldots$ implies that $H \equiv 0$, and hence $H^{(k)} \equiv 0$, which contradicts $H^{(k)} \equiv \psi \not \equiv 0$.

Lemma 3.5 Let $k$ and $l$ be positive integers, and let $R$ be a rational function. If $R^{(k)}(z) \neq z^{l}$ for all $z \in \mathbb{C}$, then

$$
R(z)=\frac{\prod_{i=1}^{n+1+l}\left(z-\alpha_{i}\right)}{(l+k)(l+k-1) \cdots(l+1)(z-\beta)^{n+1-k}},
$$

where $n(\geq k-1)$ is an integer and $\alpha_{i}, \beta \in \mathbb{C}(1 \leq i \leq l+n+1)$.

Proof Obviously, $\left(R^{(k-1)}(z)-\frac{z^{l+1}}{l+1}\right)^{\prime} \neq 0$. Then $R^{(k-1)}(z)-\frac{z^{l+1}}{l+1}$ is a nonconstant rational function. By Lemma 2.7,

$$
R^{(k-1)}(z)=\frac{z^{l+1}}{l+1}+a z+b \quad \text { or } \quad R^{(k-1)}(z)=\frac{z^{l+1}}{l+1}+\frac{a}{(z+c)^{n}}+b
$$

where $n(\geq k)$ is an integer and $a(\neq 0), b, c \in \mathbb{C}$. In fact, if $R^{(k-1)}(z)=\frac{z^{l+1}}{l+1}+\frac{a}{(z+c)^{n}}+b$, then $z=-c$ is a pole of $R(z)$ of order at least $k$, and hence $n \geq k$. Now, we have

$$
R(z)=\frac{z^{l+k}}{(l+k) \cdots(l+1)}+P_{k}(z) \quad \text { or } \quad R(z)=\frac{z^{l+k}}{(l+k) \cdots(l+1)}+\frac{c_{1}}{(z+c)^{n+1-k}}+P_{k-1}(z)
$$

where $P_{k}(z)$ is a polynomial of degree $k, P_{k-1}(z)$ is a polynomial of degree at most $k-1$, and $c_{1}$ is a constant. Thus $R(z)$ has the following form:

$$
R(z)=\frac{\prod_{i=1}^{n+1+l}\left(z-\alpha_{i}\right)}{(l+k)(l+k-1) \cdots(l+1)(z-\beta)^{n+1-k}},
$$

where $n(\geq k-1)$ is an integer and $\alpha_{i}, \beta \in \mathbb{C}(1 \leq i \leq l+n+1)$.

Lemma 3.6 Let $d$ and $k(\geq 1)$ be integers, and let $f$ be a transcendental meromorphic function, all of whose zeros have multiplicity at least $k+1$. Set $g(z):=\frac{f(z)}{z^{d}}$ with $g:=f$ if 
$d=0$. If $\overline{\lim }_{r \rightarrow \infty} \frac{T(r, f)}{r^{2}}=\infty$, then there exist points $a_{n} \rightarrow \infty$ and positive numbers $\delta_{n} \rightarrow 0$ such that

$$
\frac{f\left(a_{n}\right)}{a_{n}^{d}} \rightarrow 0, \quad \frac{f^{(k)}\left(a_{n}\right)}{a_{n}^{d}} \rightarrow \infty \quad \text { and } \quad S\left(\Delta\left(a_{n}, \delta_{n}\right), g\right) \rightarrow \infty .
$$

Proof By standard results in Nevanlinna theory, $T(r, f)=T\left(r, z^{d} g\right) \leq T(r, g)+T\left(r, z^{d}\right)$ and $T\left(r, z^{d}\right)=O(\log r)$ as $r \rightarrow \infty$. Thus, $\overline{\lim }_{r \rightarrow \infty} \frac{T(r, g)}{r^{2}}=\infty$, and then $\overline{\lim }_{r \rightarrow \infty} \frac{T_{0}(r, g)}{r^{2}}=\infty$.

We claim that there exist $t_{n} \rightarrow \infty$ and $\varepsilon_{n} \rightarrow 0$ such that

$$
S\left(\Delta\left(t_{n}, \varepsilon_{n}\right), g\right)=\frac{1}{\pi} \iint_{\left|z-t_{n}\right|<\varepsilon_{n}}\left[g^{\#}(z)\right]^{2} \mathrm{~d} x \mathrm{~d} y \rightarrow \infty .
$$

Otherwise there would exist $\varepsilon>0$ and $M>0$ such that $S\left(\Delta\left(z_{0}, \varepsilon\right), g\right)<M$ for all $z_{0} \in \mathbb{C}$. From this follows

$$
S(r, g)=\frac{1}{\pi} \iint_{|z|<r}\left[g^{\#}(z)\right]^{2} \mathrm{~d} x \mathrm{~d} y=O\left(r^{2}\right) .
$$

Obviously, $T_{0}(r, g)=\int_{0}^{r} \frac{S(t)}{t} \mathrm{~d} t=O\left(r^{2}\right)$. This contradicts the fact that $\varlimsup_{r \rightarrow \infty} \frac{T_{0}(r, g)}{r^{2}}=\infty$.

By (3.4), there exist points $b_{n}$ such that $\left|b_{n}-t_{n}\right|<\varepsilon_{n}$ and $g^{\#}\left(b_{n}\right) \rightarrow \infty$. Set $g_{n}(z):=g(z+$ $\left.b_{n}\right)$. Clearly, $g_{n}^{\#}(0)=g^{\#}\left(b_{n}\right) \rightarrow \infty$, and hence $\left\{g_{n}\right\}$ is not normal at 0 . Obviously, all zeros of $g_{n}(z)$ have multiplicity at least $k+1$ in $\Delta$ for sufficiently large $n$. Using Lemma 2.2 for $\alpha=k-1 / 2$, there exist points $z_{n} \rightarrow 0$, positive numbers $\rho_{n} \rightarrow 0$, and a subsequence of $\left\{g_{n}\right\}$ (still denoted by $\left.\left\{g_{n}\right\}\right)$ such that $G_{n}(\zeta)=\frac{g_{n}\left(z_{n}+\rho_{n} \zeta\right)}{\rho_{n}^{k-1 / 2}} \stackrel{\chi}{\Rightarrow} G(\zeta)$ in $\mathbb{C}$, where $G$ is a nonconstant meromorphic function in $\mathbb{C}$, all of whose zeros have multiplicity at least $k+1$.

Since $G^{(k)}(\zeta)$ is not a constant (otherwise, either $G(\zeta)$ is a constant, or the zero of $G(\zeta)$ have multiplicity at most $k$ ), we may assume $\zeta_{0}$ is not a zero or pole of $G^{(k)}(\zeta)$. Set $a_{n}$ := $z_{n}+\rho_{n} \zeta_{0}+b_{n}$. Now we have

$$
g^{(i)}\left(a_{n}\right)=g_{n}^{(i)}\left(z_{n}+\rho_{n} \zeta_{0}\right)=\rho_{n}^{k-\frac{1}{2}-i} G_{n}^{(i)}\left(\zeta_{0}\right)
$$

where $i=0,1, \ldots, k$. Obviously, $a_{n} \rightarrow \infty, g^{(k)}\left(a_{n}\right) \rightarrow \infty$, and $g^{(i)}\left(a_{n}\right) \rightarrow 0$ for $i=0,1, \ldots$, $k-1$.

Now, we have $\frac{f\left(a_{n}\right)}{a_{n}^{d}}=g\left(a_{n}\right) \rightarrow 0$ and

$$
\frac{f^{(k)}\left(a_{n}\right)}{a_{n}^{d}}=\left.\frac{\left(z^{d} g(z)\right)^{(k)}}{a_{n}^{d}}\right|_{z=a_{n}}=\sum_{m=1}^{m=k} T_{m} a_{n}^{m-k} g^{(m)}\left(a_{n}\right) \rightarrow \infty,
$$

where $T_{m}(m=0,1, \ldots, k)$ are constants and $T_{k} \neq 0$. Set $\delta_{n}:=\varepsilon_{n}+\left|a_{n}-t_{n}\right|$. Obviously, $\delta_{n} \rightarrow 0$ and $\Delta\left(t_{n}, \varepsilon_{n}\right) \subset \Delta\left(a_{n}, \delta_{n}\right)$, and hence $S\left(\Delta\left(a_{n}, \delta_{n}\right), g\right) \rightarrow \infty$.

Lemma 3.7 Let $k$ be a positive integer, and let $\left\{f_{n}\right\}$ be a family of meromorphic functions in $D$, all of whose poles are multiple and whose zeros all have multiplicity at least $k+1$. Let $\left\{h_{n}\right\}$ be a family of meromorphic functions in D such that $h_{n} \stackrel{x}{\Rightarrow} h$ in $D$, where $h(\neq 0)$ is a holomorphic function in $D$. If $f_{n}^{(k)}(z) \neq h_{n}(z)$ for each $n \in \mathbb{N}$ and $z \in D$, then $\left\{f_{n}\right\}$ is normal in $D$. 
Proof Suppose that $\left\{f_{n}\right\}$ is not normal at a point $z^{*} \in D$. Then by Lemma 2.2, there exist points $z_{n} \rightarrow z^{*}$, positive numbers $\rho_{n} \rightarrow 0$ and a subsequence of $\left\{f_{n}\right\}$ (still denoted by $\left\{f_{n}\right\}$ ) such that $g_{n}(\zeta)=\frac{f_{n}\left(z_{n}+\rho_{n} \zeta\right)}{\rho_{n}^{k}} \stackrel{\chi}{\Rightarrow} g(\zeta)$ in $\mathbb{C}$, where $g$ is a nonconstant meromorphic function in $\mathbb{C}$, all of whose poles are multiple and whose zeros all have multiplicity at least $k+1$. In particular, $g$ has order at most 2. Obviously, $g_{n}^{(k)}(\zeta)=f_{n}^{(k)}\left(z_{n}+\rho_{n} \zeta\right) \neq h_{n}\left(z_{n}+\rho_{n} \zeta\right)$ and $h_{n}\left(z_{n}+\rho_{n} \zeta\right) \Rightarrow h\left(z^{*}\right)(\neq 0)$ in $\mathbb{C}$. By Lemma 3.1, we have either $g^{(k)}(z) \equiv h\left(z^{*}\right)$ or $f^{(k)}(z) \neq$ $h\left(z^{*}\right)$ in $\mathbb{C}$. Firstly, suppose that $g^{(k)}(z) \equiv h\left(z^{*}\right)$ in $\mathbb{C}$. It follows that $g(z)=a_{k} z^{k}+a_{k-1} z^{k-1}+$ $\cdots+a_{1} z+a_{0}$. We arrive at a contradiction as $g(z)$ is nonconstant and all zeros of $g(z)$ have multiplicity at least $k+1$. Secondly, suppose that $g^{(k)}(z) \neq h\left(z^{*}\right)$ in $\mathbb{C}$. By Lemma 2.12 , then $g(z)$ is a constant. A contradiction.

Lemma 3.8 Let $k$ be a positive integer, and let $\left\{f_{n}\right\}$ be a family of meromorphic functions in $D$, all of whose poles are multiple and whose zeros all have multiplicity at least $k+1$. Let $\left\{h_{n}\right\}$ be a family of meromorphic functions in D such that $h_{n} \stackrel{x}{\Rightarrow} h$ in $D$, where $h(\neq 0)$ is a meromorphic function in D. Suppose that $h$ and $h_{n}$ have the same poles, all with the same multiplicity. If $f_{n}^{(k)}(z) \neq h_{n}(z)$ for all $n \in \mathbb{N}$ and all $z \in D$, then $\left\{f_{n}\right\}$ is normal in $D$.

Lemma 3.8 can be proved by an exactly analogous argument as in the proof of Theorem 1 in [16]. To facilitate the reading, Lemma 3.8 was proved in this paper.

Lemma 3.9 Let $k \geq 2$ be an integer, and let $\left\{f_{n}\right\}$ be a family of meromorphic functions in $D$, all of whose zeros have multiplicity at least $k+1$. Let $\left\{h_{n}\right\}$ be a family of meromorphic functions in D such that $h_{n}(z) \stackrel{x}{\Rightarrow} h(z)$ in $D$, where $h(\not \equiv 0, \infty)$ is a meromorphic function. Let $E \subset D$ be a set which has no accumulation points in D. Suppose that:

(*a) $h$ and $h_{n}$ have the same zeros and poles with the same multiplicity;

(*b) for all $n \in \mathbb{N}$ and all $z \in D, f_{n}^{(k)}(z) \neq h_{n}(z)$;

(*c) for each $\tilde{a} \in E$, no subsequence of $\left\{f_{n}\right\}$ is normal at $\tilde{a}$; and

$(* \mathrm{~d}) f_{n_{k}} \stackrel{x}{\Rightarrow} f(z)$ in $D \backslash E$, where $f(z)$ is meromorphic or identically infinite there.

Then

(*e) for each $\tilde{a} \in E, h(\tilde{a}) \neq \infty$;

$(* \mathrm{f})$ for each $\tilde{a} \in E$, there exist $r_{\tilde{a}}>0$ and $N_{\tilde{a}}>0$ such that for sufficiently large $n$, $n\left(\Delta\left(\tilde{a}, r_{\tilde{a}}\right), \frac{1}{f_{n}}\right)<N_{\tilde{a}}$, where $r_{\tilde{a}}$ and $N_{\tilde{a}}$ only depend on $\tilde{a}$; and

$(* \mathrm{~g})$ for each $\tilde{a} \in E, f(z)=\int_{\tilde{a}}^{z} \int_{\tilde{a}}^{\zeta_{1}} \cdots \int_{\tilde{a}}^{\zeta_{k-1}} h\left(\zeta_{k}\right) \mathrm{d} \zeta_{k} \mathrm{~d} \zeta_{k-1} \cdots \mathrm{d} \zeta_{1}$ in $D \backslash E$.

\section{Proof of Lemma 3.8}

Proof Since normality is a local property, by Lemma 3.7, we only need to prove that $\left\{f_{n}\right\}$ is normal at every pole of $h(z)$. Making standard normalizations, we may assume $D=\Delta$ and

$$
h(z)=\frac{1}{z^{l}}+\frac{a_{-l+1}}{z^{l-1}}+\cdots=\frac{\phi(z)}{z^{l}} \quad(z \in \Delta),
$$

where $l$ is a positive integer, $\phi(0)=1$, and $\phi(z) \neq 0, \infty$ for all $z \in \Delta$.

Set $h_{n}(z):=\frac{\phi_{n}(z)}{z^{l}}$. Since $h$ and $h_{n}$ have the same poles, all with the same multiplicity and $h_{n} \stackrel{x}{\Rightarrow} h$ in $D$, we have $\phi_{n}(z) \Rightarrow \phi(z)$ in $\Delta$.

Clearly, it is enough to show that $\left\{f_{n}\right\}$ is normal at $z=0$. Suppose, on the contrary, that $\left\{f_{n}\right\}$ is not normal at 0 . By Lemma $3.7,\left\{f_{n}\right\}$ is normal in $\Delta^{\prime}$. Taking a subsequence and 
renumbering, we may assume that no subsequence of $\left\{f_{n}\right\}$ is normal at 0 . Our goal is to obtain a contradiction in the sequel.

We distinguish two cases.

Case $1.1 \leq l \leq k$.

By Lemma 2.2, there exist points $z_{n} \rightarrow 0$, positive numbers $\rho_{n} \rightarrow 0$ and a subsequence of $\left\{f_{n}\right\}$ (still denoted by $\left\{f_{n}\right\}$ ) such that

$$
F_{n}(\zeta)=\frac{f_{n}\left(z_{n}+\rho_{n} \zeta\right)}{\rho_{n}^{k-l}} \stackrel{\chi}{\Rightarrow} F(\zeta) \quad \text { in } \mathbb{C},
$$

where $F(\zeta)$ is a nonconstant meromorphic function in $\mathbb{C}$. By Hurwitz's theorem, all poles of $F(\zeta)$ are multiple and all zeros of $F(\zeta)$ have multiplicity at least $k+1$.

Taking a subsequence and renumbering, we may assume $z_{n} / \rho_{n} \rightarrow \alpha$ as $n \rightarrow \infty$, where $\alpha \in \mathbb{C}$ or $\alpha=\infty$. Again we distinguish two subcases.

Subcase 1.1. $z_{n} / \rho_{n} \rightarrow \infty$.

Set $g_{n}(\zeta):=z_{n}^{l-k} f_{n}\left(z_{n}+z_{n} \zeta\right)=z_{n}^{l-k} f_{n}\left(z_{n}(1+\zeta)\right)$. Obviously, all poles of $g_{n}(\zeta)$ are multiple and all zeros of $g_{n}(\zeta)$ have multiplicity at least $k+1$,

$$
g_{n}^{(k)}(\zeta)=z_{n}^{l} f_{n}^{(k)}\left(z_{n}(1+\zeta)\right) \neq \frac{\phi_{n}\left(z_{n}(1+\zeta)\right)}{(1+\zeta)^{l}} \quad \text { and } \quad \frac{\phi_{n}\left(z_{n}(1+\zeta)\right)}{(1+\zeta)^{l}} \quad \stackrel{\chi}{\Rightarrow} \frac{1}{(1+\zeta)^{l}} \quad \text { in } \mathbb{C} .
$$

Then, by Lemma 3.7, the family $\left\{g_{n}\right\}$ is normal in $\Delta$. Taking a subsequence and renumbering, we may assume that $g_{n}(\zeta) \stackrel{\not}{\Rightarrow} g(\zeta)$ in $\Delta$. Obviously, all zeros of $g(\zeta)$ have multiplicity at least $k+1$ in $\Delta$.

We claim that $g(\zeta)$ is a meromorphic function in $\Delta$. Otherwise, suppose that $g(\zeta) \equiv \infty$ in $\Delta$. Then

$$
\begin{aligned}
F_{n}(\zeta) & =\frac{f_{n}\left(z_{n}+z_{n}\left(\frac{\rho_{n}}{z_{n}} \zeta\right)\right)}{\rho_{n}^{k-l}}=\left(\frac{z_{n}}{\rho_{n}}\right)^{k-l} \frac{f_{n}\left(z_{n}+z_{n}\left(\frac{\rho_{n}}{z_{n}} \zeta\right)\right)}{z_{n}^{k-l}} \\
& =\left(\frac{z_{n}}{\rho_{n}}\right)^{k-l} g_{n}\left(\frac{\rho_{n}}{z_{n}} \zeta\right) \stackrel{\chi}{\Rightarrow} \infty \text { in } \mathbb{C} .
\end{aligned}
$$

Thus, $F(\zeta) \equiv \infty$ in $\mathbb{C}$. A contradiction.

We claim that $g(0) \neq \infty$. Since $F(\zeta)$ is a nonconstant meromorphic function in $\mathbb{C}$, there exist $\zeta_{0} \in \mathbb{C}$ such that $F\left(\zeta_{0}\right) \neq \infty$. Noting that $\frac{\rho_{n}}{z_{n}} \zeta_{0} \rightarrow 0$ as $n \rightarrow \infty$, we have

$$
g(0)=\lim _{n \rightarrow \infty} g_{n}\left(\frac{\rho_{n}}{z_{n}} \zeta_{0}\right)=\lim _{n \rightarrow \infty} \frac{f_{n}\left(z_{n}+\rho_{n} \zeta_{0}\right)}{\rho_{n}^{k-l}}\left(\frac{\rho_{n}}{z_{n}}\right)^{k-l}= \begin{cases}F\left(\zeta_{0}\right) & \text { if } l=k, \\ 0 & \text { if } l<k .\end{cases}
$$

For any $\zeta \in \mathbb{C} \backslash F^{-1}(\infty)$, we have

$$
\begin{aligned}
F^{(k-l)}(\zeta) & =\lim _{n \rightarrow \infty} F_{n}^{(k-l)}(\zeta)=\lim _{n \rightarrow \infty} f_{n}^{(k-l)}\left(z_{n}+\rho_{n} \zeta\right)=\lim _{n \rightarrow \infty} f_{n}^{(k-l)}\left(z_{n}+z_{n}\left(\frac{\rho_{n}}{z_{n}} \zeta\right)\right) \\
& =\lim _{n \rightarrow \infty} g_{n}^{(k-l)}\left(\frac{\rho_{n}}{z_{n}} \zeta\right)=g^{(k-l)}(0) .
\end{aligned}
$$

This implies that $F^{(k-l)}(\zeta) \equiv g^{(k-l)}(0)$ in $\mathbb{C} \backslash F^{-1}(\infty)$ and thus $F^{(k)}(\zeta)$ is a constant in $\mathbb{C}$. It follows that $F(\zeta)=a_{k} \zeta^{k}+\cdots+a_{1} \zeta+a_{0}$. We arrive at a contradiction as $F(\zeta)$ is nonconstant and all zeros of $F(\zeta)$ have multiplicity at least $k+1$ in $\mathbb{C}$. 
Subcase 1.2. $z_{n} / \rho_{n} \rightarrow \alpha(\alpha \in \mathbb{C})$.

Clearly, we have

$$
\begin{aligned}
& F_{n}^{(k)}(\zeta)=\rho_{n}^{l} f_{n}^{(k)}\left(z_{n}+\rho_{n} \zeta\right) \neq \rho_{n}^{l} h_{n}\left(z_{n}+\rho_{n} \zeta\right) \quad \text { and } \\
& \rho_{n}^{l} h_{n}\left(z_{n}+\rho_{n} \zeta\right)=\frac{\rho_{n}^{l} \phi_{n}\left(z_{n}+\rho_{n} \zeta\right)}{\left(z_{n}+\rho_{n} \zeta\right)^{l}} \stackrel{\chi}{\Rightarrow} \frac{1}{(\alpha+\zeta)^{l}} \quad \text { in } \mathbb{C} .
\end{aligned}
$$

By Lemma 3.1, either $F^{(k)}(\zeta) \neq 1 /(\alpha+\zeta)^{l}$ or $F^{(k)}(\zeta) \equiv 1 /(\alpha+\zeta)^{l}$ in $\mathbb{C}$. The latter possibility contradicts the fact that all poles of $F^{(k)}(\zeta)$ have multiplicity at least $k+2(>l)$. Thus $F^{(k)}(\zeta) \neq 1 /(\alpha+\zeta)^{l}$ in $\mathbb{C}$. It follows from Lemma 2.8 and Lemma 2.9 that $F(\zeta)$ is a constant. A contradiction.

Case $2 . l \geq k+1$.

Set

$$
\mathcal{G}:=\left\{g_{n}(z) \mid g_{n}(z)=f_{n}(z) / h_{n}(z), z \in \Delta\right\} .
$$

Clearly, all poles of $g_{n}(z)$ are multiple and all zeros of $g_{n}(z)$ have multiplicity at least $k+1$ in $\Delta$. Since $f_{n}^{(k)}(0) \neq h_{n}(0)=\infty$, we have $f_{n}(0) \neq \infty$. Thus, for each $n, g_{n}(0)=f_{n}(0) / h_{n}(0)=0$. Obviously, $g_{n}$ has a zero of order at least $l$ at $z=0$ for each $n$.

We first prove that $\mathcal{G}$ is normal in $\Delta$. Suppose that $\mathcal{G}$ is not normal at $z_{0} \in \Delta$. Then by Lemma 2.2, there exist points $z_{n} \rightarrow z_{0}$, positive numbers $\rho_{n} \rightarrow 0$, and a subsequence of $\left\{g_{n}\right\}$ (still denoted by $\left\{g_{n}\right\}$ ) such that

$$
G_{n}(\zeta)=\frac{g_{n}\left(z_{n}+\rho_{n} \zeta\right)}{\rho_{n}^{k}} \stackrel{\chi}{\Rightarrow} G(\zeta) \quad \text { in } \mathbb{C},
$$

where $G(\zeta)$ is a nonconstant meromorphic function in $\mathbb{C}$. In particular, $G(\zeta)$ has order at most 2. By Hurwitz's theorem, all poles of $G(\zeta)$ are multiple and all zeros of $G(\zeta)$ have multiplicity at least $k+1$.

Taking a subsequence and renumbering, we may assume $z_{n} / \rho_{n} \rightarrow \alpha$ as $n \rightarrow \infty$, where $\alpha \in \mathbb{C}$ or $\alpha=\infty$. Again we distinguish two subcases.

Subcase 2.1. $z_{n} / \rho_{n} \rightarrow \infty$.

By simple calculation, we have

$$
g_{n}^{(k)}(z)=\frac{f_{n}^{(k)}(z)}{h_{n}(z)}-\left(\begin{array}{c}
n \\
1
\end{array}\right) g_{n}^{(k-1)}(z) \frac{h_{n}^{\prime}(z)}{h_{n}(z)}-\left(\begin{array}{c}
n \\
2
\end{array}\right) g_{n}^{(k-2)}(z) \frac{h_{n}^{\prime \prime}(z)}{h_{n}(z)}-\cdots-g_{n}(z) \frac{h_{n}^{(k)}(z)}{h_{n}(z)} .
$$

Then we have

$$
\begin{aligned}
G_{n}^{(k)}(\zeta)= & g_{n}^{(k)}\left(z_{n}+\rho_{n} \zeta\right) \\
= & \frac{f_{n}^{(k)}\left(z_{n}+\rho_{n} \zeta\right)}{h_{n}\left(z_{n}+\rho_{n} \zeta\right)}-\left(\begin{array}{c}
n \\
1
\end{array}\right) g_{n}^{(k-1)}\left(z_{n}+\rho_{n} \zeta\right) \frac{h_{n}^{\prime}\left(z_{n}+\rho_{n} \zeta\right)}{h_{n}\left(z_{n}+\rho_{n} \zeta\right)}-\cdots \\
& -g_{n}\left(z_{n}+\rho_{n} \zeta\right) \frac{h_{n}^{(k)}\left(z_{n}+\rho_{n} \zeta\right)}{h_{n}\left(z_{n}+\rho_{n} \zeta\right)} \\
= & \frac{f_{n}^{(k)}\left(z_{n}+\rho_{n} \zeta\right)}{h_{n}\left(z_{n}+\rho_{n} \zeta\right)}-\left(\begin{array}{c}
n \\
1
\end{array}\right) g_{n}^{(k-1)}\left(z_{n}+\rho_{n} \zeta\right)\left(\frac{-l}{z_{n}+\rho_{n} \zeta}+\frac{\phi_{n}^{\prime}\left(z_{n}+\rho_{n} \zeta\right)}{\phi_{n}\left(z_{n}+\rho_{n} \zeta\right)}\right)-\cdots
\end{aligned}
$$




$$
\begin{aligned}
& -g_{n}\left(z_{n}+\rho_{n} \zeta\right)\left(\frac{(-1)^{k} l(l-1) \cdots(l-k+1)}{\left(z_{n}+\rho_{n} \zeta\right)^{k}}\right. \\
& \left.+\left(\begin{array}{c}
k \\
1
\end{array}\right) \frac{(-1)^{k-1} l(l-1) \cdots(l-k+2)}{\left(z_{n}+\rho_{n} \zeta\right)^{k-1}} \frac{\phi_{n}^{\prime}\left(z_{n}+\rho_{n} \zeta\right)}{\phi_{n}\left(z_{n}+\rho_{n} \zeta\right)}+\cdots+\frac{\phi_{n}^{(k)}\left(z_{n}+\rho_{n} \zeta\right)}{\phi_{n}\left(z_{n}+\rho_{n} \zeta\right)}\right) \\
= & \frac{f_{n}^{(k)}\left(z_{n}+\rho_{n} \zeta\right)}{h_{n}\left(z_{n}+\rho_{n} \zeta\right)}-\left(\begin{array}{l}
n \\
1
\end{array}\right) \frac{g_{n}^{(k-1)}\left(z_{n}+\rho_{n} \zeta\right)}{\rho_{n}}\left(\frac{-l}{z_{n} / \rho_{n}+\zeta}+\frac{\rho_{n} \phi_{n}^{\prime}\left(z_{n}+\rho_{n} \zeta\right)}{\phi_{n}\left(z_{n}+\rho_{n} \zeta\right)}\right)-\cdots \\
& -\frac{g_{n}\left(z_{n}+\rho_{n} \zeta\right)}{\rho_{n}^{k}}\left(\frac{(-1)^{k} l(l-1) \cdots(l-k+1)}{\left(z_{n} / \rho_{n}+\zeta\right)^{k}}\right. \\
& \left.+\left(\begin{array}{l}
k \\
1
\end{array}\right) \frac{(-1)^{k-1} l(l-1) \cdots(l-k+2)}{\left(z_{n} / \rho_{n}+\zeta\right)^{k-1}} \frac{\rho_{n} \phi_{n}^{\prime}\left(z_{n}+\rho_{n} \zeta\right)}{\phi_{n}\left(z_{n}+\rho_{n} \zeta\right)}+\cdots+\frac{\rho_{n}^{k} \phi_{n}^{(k)}\left(z_{n}+\rho_{n} \zeta\right)}{\phi_{n}\left(z_{n}+\rho_{n} \zeta\right)}\right) .
\end{aligned}
$$

On the other hand, we have $\lim _{n \rightarrow \infty} \frac{1}{z_{n} / \rho_{n}+\zeta}=0$ and then for $i=1,2, \ldots, k$,

$$
\frac{\rho_{n} \phi_{n}^{(i)}\left(z_{n}+\rho_{n} \zeta\right)}{\phi_{n}\left(z_{n}+\rho_{n} \zeta\right)} \Rightarrow 0 \quad \text { in } \mathbb{C}
$$

Since $g_{n}\left(z_{n}+\rho_{n} \zeta\right) / \rho_{n}^{k} \stackrel{\chi}{\Rightarrow} G(\zeta), g_{n}^{(k-i)}(\hat{\zeta}) / \rho_{n}^{i}$ is locally bounded in $\mathbb{C} \backslash G^{-1}(\infty)$. Thus,

$$
\frac{f_{n}^{(k)}\left(z_{n}+\rho_{n} \zeta\right)}{h_{n}\left(z_{n}+\rho_{n} \zeta\right)} \quad \stackrel{x}{\Rightarrow} \quad G^{(k)}(\zeta) \quad \text { in } \mathbb{C} \backslash G^{-1}(\infty)
$$

Since $f_{n}^{(k)}(z) / h_{n}(z) \neq 1$, Hurwitz's theorem yields that either $G^{(k)}(\zeta) \neq 1$ or $G^{(k)}(\zeta) \equiv 1$ in $\mathbb{C}$. If $G^{(k)}(\zeta) \neq 1$ in $\mathbb{C}$, then by Lemma $2.12, G(\zeta)$ is a constant. A contradiction. Thus $G^{(k)}(\zeta) \equiv 1$, and then $G(\zeta)=\frac{1}{k !} \zeta^{k}+c_{k-1} \zeta^{k-1}+\cdots+c_{0}$. This contradicts the fact that all zeros of $G(\zeta)$ have multiplicity at least $k+1$.

Subcase 2.2. $z_{n} / \rho_{n} \rightarrow \alpha(\alpha \in \mathbb{C})$.

$$
\frac{g_{n}\left(\rho_{n} \zeta\right)}{\rho_{n}^{k}}=\frac{g_{n}\left(z_{n}+\rho_{n}\left(\zeta-z_{n} / \rho_{n}\right)\right)}{\rho_{n}^{k}}=G_{n}\left(\zeta-z_{n} / \rho_{n}\right) \quad \stackrel{\chi}{\Rightarrow} G(\zeta-\alpha)=\widehat{G}(\zeta) \quad \text { in } \mathbb{C} .
$$

Clearly, all poles of $\widehat{G}(\zeta)$ are multiple and all zeros of $\widehat{G}(\zeta)$ have multiplicity at least $k+1$, and $\widehat{G}(\zeta)$ has a zero of order at least $l$ at $\zeta=0$.

Set $H_{n}(\zeta):=\rho_{n}^{l-k} f_{n}\left(\rho_{n} \zeta\right)$. Then, we have

$$
H_{n}(\zeta)=\rho_{n}^{l} h_{n}\left(\rho_{n} \zeta\right) \frac{f_{n}\left(\rho_{n} \zeta\right)}{\rho_{n}^{k} h_{n}\left(\rho_{n} \zeta\right)}=\rho_{n}^{l} h_{n}\left(\rho_{n} \zeta\right) \frac{g_{n}\left(\rho_{n} \zeta\right)}{\rho_{n}^{k}}
$$

Noting that $\rho_{n}^{l} h_{n}\left(\rho_{n} \zeta\right) \stackrel{\chi}{\Rightarrow} \frac{1}{\zeta^{l}}$ in $\mathbb{C}$, we have

$$
H_{n}(\zeta) \stackrel{\chi}{\Rightarrow} \quad \frac{1}{\zeta^{l}} \widehat{G}(\zeta)=H(\zeta) \quad \text { in } \mathbb{C} \backslash\{0\}
$$

Since $\widehat{G}(\zeta)$ has a zero at $\zeta=0$, by $(4.1)$, there exists $\delta \in(0,1)$ such that $g_{n}\left(\rho_{n} \zeta\right)$ is holomorphic in $\Delta(0, \delta)$ for sufficiently large $n$, and thus $H_{n}(\zeta)$ is holomorphic in $\Delta(0, \delta)$ for sufficiently large $n$. By the maximum principle, we have

$$
H_{n}(\zeta) \stackrel{\chi}{\Rightarrow} \quad \frac{1}{\zeta^{l}} \widehat{G}(\zeta)=H(\zeta) \quad \text { in } \mathbb{C}
$$


Obviously, all poles of $H(\zeta)$ are multiple and all zeros of $H(\zeta)$ have multiplicity at least $k+1$. Since $\widehat{G}(\zeta)$ has a zero of order at least $l$ at $\zeta=0$, we have $H(0) \neq \infty$ and thus $H(\zeta)$ is a meromorphic function in $\mathbb{C}$. Noting that

$$
H_{n}^{(k)}(\zeta)=\rho_{n}^{l} f_{n}^{(k)}\left(\rho_{n} \zeta\right) \neq \rho_{n}^{l} h_{n}\left(\rho_{n} \zeta\right) \quad \text { and } \quad \rho_{n}^{l} h_{n}\left(\rho_{n} \zeta\right) \quad \stackrel{\chi}{\Rightarrow} \quad \frac{1}{\zeta^{l}} \quad \text { in } \mathbb{C}
$$

By Lemma 3.1, either $H^{(k)}(\zeta) \neq 1 / \zeta^{l}$ or $H^{(k)}(\zeta) \equiv 1 / \zeta^{l}$ in $\mathbb{C}$. The latter possibility contradicts the fact that $H(0) \neq \infty$. Thus $H^{(k)}(\zeta) \neq 1 / \zeta^{l}$ in $\mathbb{C}$. It follows from Lemma 2.8 and Lemma 2.9 that $H(\zeta)$ is a constant. Next we will show that this is impossible. Indeed, suppose that $H \equiv c$. Since $G$ is not a constant, $c \neq 0$. Then we have

$$
f_{n}(0)=\frac{H_{n}(0)}{\rho_{n}^{l-k}} \rightarrow \infty \quad \text { as } n \rightarrow \infty
$$

Suppose first that there exists $\delta \in(0,1)$ such that $f_{n}(z) \neq 0$ in $\Delta(0, \delta)$ for sufficiently large $n$. By Lemma 3.3, $\left\{f_{n}\right\}$ is normal in $\Delta(0, \delta)$. But this contradicts our assumption that no subsequence of $\left\{f_{n}\right\}$ is normal at 0 . Hence, taking a subsequence and renumbering, we may assume that $z_{n}^{*}$ is the zero of $f_{n}$ of smallest modulus and $z_{n}^{*} \rightarrow 0$. Since $H_{n}(\zeta)=\rho_{n}^{l-k} f_{n}\left(\rho_{n} \zeta\right) \Rightarrow c(\neq 0)$, we have $z_{n}^{*} / \rho_{n} \rightarrow \infty$. Set

$$
H_{n}^{*}(\zeta):=\left(z_{n}^{*}\right)^{l-k} f_{n}\left(z_{n}^{*} \zeta\right)
$$

In view of the fact that

$$
H_{n}^{*(k)}(\zeta)=\left(z_{n}^{*}\right)^{l} f_{n}^{(k)}\left(z_{n}^{*} \zeta\right) \neq \frac{\phi_{n}\left(z_{n}^{*} \zeta\right)}{\zeta^{l}} \stackrel{\chi}{\Rightarrow} \frac{1}{\zeta^{l}}
$$

and $H_{n}^{*}(\zeta) \neq 0$ in $\Delta$, by Lemma $3.3,\left\{H_{n}^{*}(\zeta)\right\}$ is normal in $\Delta$. By Lemma 3.7, $\left\{H_{n}^{*}(\zeta)\right\}$ is normal in $\mathbb{C} \backslash\{0\}$. Hence, $\left\{H_{n}^{*}(\zeta)\right\}$ is normal in $\mathbb{C}$. Therefore, there exists a subsequence $\left\{H_{n}^{*}(\zeta)\right\}$ (still denoted by $\left.\left\{H_{n}^{*}(\zeta)\right\}\right)$ such that $H_{n}^{*} \stackrel{\chi}{\Rightarrow} H^{*}$ in $\mathbb{C}$. By the definition of $H_{n}^{*}(\zeta)$, we get $H_{n}^{*}(1)=0$ and thus $H^{*}(1)=0$. Since

$$
H_{n}^{*}(0)=H_{n}(0)\left(\frac{z_{n}^{*}}{\rho_{n}}\right)^{l-k}=c\left(\frac{z_{n}^{*}}{\rho_{n}}\right)^{l-k} \rightarrow \infty,
$$

we get that $H^{*}(0)=\infty$. Thus $H^{*}$ is nonconstant. However, since $H^{*}(0)=\infty$ and $H_{n}^{*}(\zeta) \neq 0$ in $\Delta$, we have $H^{*} \neq 0$ in $\Delta$ by Hurwitz's theorem. However, Lemma 2.10 implies that $H^{*}(\zeta)$ has a zero in $\Delta$. A contradiction.

Thus $\mathcal{G}$ is normal in $\Delta$. It remains to show that $\left\{f_{n}\right\}$ is normal at 0 . Since $\mathcal{G}$ is normal in $\Delta$, then $\mathcal{G}$ is equicontinuous in $\Delta$ with respect to the spherical distance. On the other hand, $g_{n}(0)=0$ for each $n$, so there exists $\delta>0$ such that $\left|g_{n}(z)\right| \leq 1$ for all $n$ in $\Delta(0, \delta)$. It follows that $f_{n}(z)$ is holomorphic in $\Delta(0, \delta)$ for all $n$. Since $\left\{f_{n}\right\}$ is normal in $\Delta^{\prime}$, there exists a subsequence of $\left\{f_{n}\right\}$ (still denoted by $\left\{f_{n}\right\}$ ) which converges locally uniformly in $\Delta^{\prime}(0, \delta)$. The maximum modulus principle implies that $f_{n}$ converges locally uniformly in $\Delta(0, \delta)$, and thus $\left\{f_{n}\right\}$ normal at $z=0$, which contradicts our assumption that no subsequence of $\left\{f_{n}\right\}$ is normal at 0 . 


\section{Proof of Lemma 3.9}

Proof It suffices to prove that each subsequence of $\left\{f_{n}\right\}$ has a subsequence which satisfies that $(* \mathrm{f})$, and prove that $(* \mathrm{e})$ and $(* \mathrm{~g})$ hold. So suppose that we have a subsequence of $\left\{f_{n}\right\}$, which (to avoid complication in notation) we again call $\left\{f_{n}\right\}$.

Without loss of generality, for each $\tilde{a} \in E$, we may assume that $\tilde{a}=0, \Delta \subset D, \Delta^{\prime} \cap E=\emptyset$, and

$$
h(z)=z^{l}+a_{l+1} z^{l+1}+\cdots=z^{l} \hat{h}(z)
$$

in $\Delta$, where $\hat{h}(0)=1$ and $\hat{h}(z) \neq 0, \infty$ in $\Delta$.

We consider the following three cases.

Case $1 . h(0)=\infty$.

We will derive a contradiction in the case, and hence $(* \mathrm{e})$ holds. For convenience, we set $m:=-l$, and then

$$
h(z)=\frac{1}{z^{m}}+\frac{a_{-m+1}}{z^{m-1}}+\cdots=\frac{\hat{h}(z)}{z^{m}}
$$

in $\Delta$, where $\hat{h}(z) \neq 0, \infty$ in $\Delta, \hat{h}(0)=1$, and $m$ is a positive integer. Clearly, we have $h(z) \neq$ $0, \infty$ in $\Delta^{\prime}, h_{n}(z) \neq 0, \infty$ in $\Delta^{\prime}$, and $h(0)=h_{n}(0)=\infty$.

Subcase 1.1. For sufficiently large $n, f_{n}(0) \neq 0$.

We claim that for each $\delta>0$, there exists at least one zero of $f_{n}$ in $\Delta(0, \delta)$ for sufficiently large $n$. Otherwise, there exist $\delta^{*}(>0)$ and a subsequence of $\left\{f_{n}\right\}$ (still denoted by $\left\{f_{n}\right\}$ ) such that $f_{n}(z) \neq 0$ in $\Delta\left(0, \delta^{*}\right)$. By Lemma $3.3,\left\{f_{n}\right\}$ is normal at 0 . This contradicts $(* \mathrm{c})$.

Taking a subsequence and renumbering, we may assume that $a_{n}(\neq 0)$ is the zero of $\left\{f_{n}\right\}$ of smallest modulus and $a_{n} \rightarrow 0$. Set $F_{n}(\zeta):=a_{n}^{m-k} f_{n}\left(a_{n} \zeta\right)$. We have:

(A1) $F_{n}(\zeta) \neq 0$ in $\Delta$;

(A2) all zeros of $F_{n}(\zeta)$ have multiplicity at least $k+1$ and $F_{n}(1)=0$;

(A3) $F_{n}^{(k)}(\zeta) \neq a_{n}^{m} h_{n}\left(a_{n} \zeta\right)$ and $a_{n}^{m} h_{n}\left(a_{n} \zeta\right) \stackrel{\chi}{\Rightarrow} \frac{1}{\zeta^{m}}$ in $\mathbb{C}$.

By Lemma 3.3 and Lemma 3.4, $\left\{F_{n}(\zeta)\right\}$ is normal in $\Delta$ and quasinormal in $\mathbb{C}$. Thus, there exist a subsequence of $\left\{F_{n}(\zeta)\right\}$ (still denoted by $\left.\left\{F_{n}(\zeta)\right\}\right)$ and $D_{1} \subset \mathbb{C}$ such that:

(B1) $D_{1}$ has no accumulation points in $\mathbb{C}$;

(B2) for each $\zeta_{0} \in D_{1}$, no subsequence of $\left\{F_{n}(\zeta)\right\}$ is normal at $\zeta_{0}$;

(B3) $F_{n}(\zeta) \stackrel{x}{\Rightarrow} F(\zeta)$ in $\mathbb{C} \backslash D_{1}$.

Obviously, $D_{1} \cap \Delta=\emptyset$ and all zeros of $F(\zeta)$ have multiplicity at least $k+1$ in $\mathbb{C} \backslash D_{1}$.

Subcase 1.1.1. $1 \notin D_{1}$.

Obviously, $F(1)=F^{(k)}(1)=0$ by (A2). Let $\zeta_{0} \in D_{1}$. By Lemma $2.5, F^{(k)}(\zeta)=\frac{1}{\zeta^{m}}$, which contradicts $F^{(k)}(1)=0$. Thus $D_{1}$ is an empty set. Since $F(1)=0, F(\zeta)$ is a meromorphic function in $\mathbb{C}$. By Lemma 3.1 and (A3), either $F^{(k)}(\zeta) \equiv \frac{1}{\zeta^{m}}$ or $F^{(k)}(\zeta) \neq \frac{1}{\zeta^{m}}$ in $\mathbb{C}$. If $F^{(k)}(\zeta) \equiv$ $\frac{1}{\zeta^{m}}$, then $F^{(k)}(1)=1$, which contradicts $F^{(k)}(1)=0$. If $F^{(k)}(\zeta) \neq \frac{1}{\zeta^{m}}$, then by Lemma 2.8 and Lemma 2.9, $F$ is a constant function. Since $F(1)=0, F(\zeta) \equiv 0$ in $\mathbb{C}$. Now,

$$
F_{n}(\zeta)=a_{n}^{m-k} f_{n}\left(a_{n} \zeta\right) \quad \Rightarrow \quad 0 \quad \text { in } \mathbb{C}
$$

We claim that for each $\delta>0$, there exists at least one pole of $f_{n}$ in $\Delta^{\prime}(0, \delta)$ for sufficiently large $n$. Otherwise, there exist $\delta^{*}(>0)$ and a subsequence of $\left\{f_{n}\right\}$ (still denoted by $\left\{f_{n}\right\}$ ) such 
that $f_{n}(z)$ has no poles in $\Delta^{\prime}\left(0, \delta^{*}\right)$. Since $f_{n}^{(k)}(z) \neq h_{n}(z)$ and $h_{n}(0)=\infty$, we have $f(0) \neq \infty$. Thus $f_{n}(z) \neq \infty$ in $\Delta\left(0, \delta^{*}\right)$. By Lemma $3.8,\left\{f_{n}\right\}$ is normal at 0 . This contradicts $(* \mathrm{c})$.

Taking a subsequence and renumbering, we may assume that $y_{n}(\neq 0)$ is the pole of $f_{n}(z)$ of smallest modulus and $y_{n} \rightarrow 0$. By Hurwitz's theorem and (5.1), $\frac{a_{n}}{y_{n}} \rightarrow 0$. Set $G_{n}(\zeta):=$ $y_{n}^{m-k} f_{n}\left(y_{n} \zeta\right)$, we have:

(C1) $G_{n}(\zeta)$ is holomorphic in $\Delta$;

(C2) $G_{n}(1)=\infty$;

(C3) all zeros of $G_{n}(\zeta)$ have multiplicity at least $k+1$;

(C4) $G_{n}^{(k)}(\zeta) \neq y_{n}^{m} h_{n}\left(y_{n} \zeta\right)$ and $y_{n}^{m} h_{n}\left(y_{n} \zeta\right) \stackrel{x}{\Rightarrow} \frac{1}{\zeta^{m}}$ in $\mathbb{C}$.

By Lemma 3.8 and Lemma 3.4, $\left\{G_{n}(\zeta)\right\}$ is normal in $\Delta$ and quasinormal in $\mathbb{C}$. Thus, there exist a subsequence of $\left\{G_{n}(\zeta)\right\}$ (still denoted by $\left.\left\{G_{n}(\zeta)\right\}\right)$ and $D_{2} \subset \mathbb{C}$ such that:

(D1) $D_{2}$ has no accumulation points in $\mathbb{C}$;

(D2) for each $\zeta_{0} \in D_{2}$, no subsequence of $\left\{G_{n}(\zeta)\right\}$ is normal at $\zeta_{0}$;

(D3) $G_{n}(\zeta) \stackrel{\not}{\Rightarrow} G(\zeta)$ in $\mathbb{C} \backslash D_{2}$.

Obviously, $D_{2} \cap \Delta=\emptyset$ and all zeros of $G(\zeta)$ have multiplicity at least $k+1$ in $\mathbb{C} \backslash D_{2}$.

Clearly, $G(0)=\lim _{n \rightarrow \infty} G_{n}\left(\frac{a_{n}}{y_{n}}\right)=y_{n}^{m-k} f_{n}\left(a_{n}\right)=0$, so $G(z)$ is meromorphic in $\mathbb{C} \backslash D_{2}$. By Lemma 3.1 and (C4), either $G^{(k)}(\zeta) \equiv \frac{1}{\zeta^{m}}$ or $G^{(k)}(\zeta) \neq \frac{1}{\zeta^{m}}$ in $\mathbb{C} \backslash D_{2}$.

Subcase 1.1.1.1. $D_{2}$ is an empty set.

By $(\mathrm{C} 2)$, we have $G(1)=\infty$. If $G^{(k)}(\zeta) \neq \frac{1}{\zeta^{m}}$ in $\mathbb{C}$, then by Lemma 2.8 and Lemma 2.9, $G(\zeta)$ is a constant function which contradicts that $G(1)=\infty$. If $G^{(k)}(\zeta)-\frac{1}{\zeta^{m}} \equiv 0$ in $\mathbb{C}$, then $G(0)=\infty$, which contradicts $G(0)=0$.

Subcase 1.1.1.2. $D_{2}$ is not an empty set.

Let $\zeta_{0} \in E$. Since $D_{2} \cap \Delta=\emptyset$, by Lemma 2.5 , we have $G^{(k)}(\zeta)=\frac{1}{\zeta^{m}}$ in $\mathbb{C} \backslash D_{2}-\{0\}$ Clearly, $G^{(k)}(\zeta)$ and $\frac{1}{\zeta^{m}}$ are meromorphic functions in $\mathbb{C} \backslash D_{2}$, so we have $G^{(k)}(\zeta)=\frac{1}{\zeta^{m}}$ in $\mathbb{C} \backslash D_{2}$, which contradicts $G(0)=0$.

Subcase 1.1.2. $1 \in D_{1}$.

By Lemma 2.5, we have $F^{(k)}(\zeta)=\frac{1}{\xi^{m}}$ in $\mathbb{C} \backslash D_{1}$. If $m \leq k$, then $F(\zeta)$ is a multi-valued function in $\mathbb{C} \backslash D_{1}$. A contradiction. Thus we have $m>k$.

We claim that $D_{1}=\{1\}$. Otherwise, there exists $\zeta_{0}$ such that $\zeta_{0} \in D_{1}$ and $\zeta_{0} \neq 0,1$. By Lemma 2.5,

$$
\begin{aligned}
& F^{(k-1)}(\zeta)=\int_{1}^{\zeta} \frac{1}{\xi^{m}} \mathrm{~d} \xi=\frac{\zeta^{-m+1}-1}{-m+1} ; \\
& F^{(k-2)}(\zeta)=\int_{1}^{\zeta} \frac{\xi^{-m+1}-1}{-m+1} \mathrm{~d} \xi=\frac{\zeta^{-m+2}}{(-m+2)(-m+1)}-\frac{\zeta}{-m+1}+\frac{1}{(-m+2)} ; \\
& F^{(k-1)}(\zeta)=\int_{\zeta_{0}}^{\zeta} \frac{1}{\xi^{m}} \mathrm{~d} \xi=\frac{\zeta^{-m+1}-\zeta_{0}^{-m+1}}{-m+1} ; \\
& F^{(k-2)}(\zeta)=\int_{\zeta_{0}}^{\zeta} \frac{\xi^{-m+1}-1}{-m+1} \mathrm{~d} \xi=\frac{\zeta^{-m+2}}{(-m+2)(-m+1)}-\frac{\zeta}{-m+1}+\frac{\zeta_{0}}{(m+2)}
\end{aligned}
$$

By (5.2) and (5.3), we obtain $\zeta_{0}=1$, which contradicts $\zeta_{0} \neq 1$. Thus $D_{1}=\{1\}$.

By Lemma 2.5,

$$
\begin{aligned}
F(\zeta) & =\int_{1}^{\zeta} \int_{1}^{\zeta 1} \cdots \int_{1}^{\zeta_{k-1}} \frac{1}{\zeta^{m}} \mathrm{~d} \zeta_{k} \mathrm{~d} \zeta_{k-1} \cdots \mathrm{d} \zeta_{1} \\
& =\frac{1+P_{k-1}(\zeta) \zeta^{m-k}}{(-m+k)(-m+k-1) \cdots(-m+1) \zeta^{m-k}}
\end{aligned}
$$


in $\mathbb{C} \backslash D_{1}$, where $P_{k-1}(\zeta)$ is a polynomial of degree $k-1$. By (5.4),

$$
F_{n}(\zeta) \stackrel{x}{\Rightarrow} \frac{1+P_{k-1}(\zeta) \zeta^{m-k}}{(-m+k)(-m+k-1) \cdots(-m+1) \zeta^{m-k}}
$$

in $\Delta$. By Hurwitz' theorem, there exist $\gamma_{n, i}(i=1,2, \ldots, m-k)$ such that $\gamma_{n, i} \rightarrow 0$ and $F_{n}\left(\gamma_{n, i}\right)=\infty$. Since $F_{n}^{(k)}(\zeta) \neq a_{n}^{m} h_{n}\left(a_{n} \zeta\right)$, we have $F_{n}(0) \neq \infty$, and hence $\gamma_{n, i} \neq 0$ for $i=$ $1,2, \ldots, m-k$.

Set $U_{n}(\xi):=s_{n}^{m-k} F_{n}\left(s_{n} \xi\right)$ and $\eta_{n, i}=\frac{\gamma_{n, i}}{s_{n}}$, where $s_{n}$ is one of $\left\{\gamma_{n, 1}, \gamma_{n, 2}, \ldots, \gamma_{n, m-k}\right\}$ of largest modulus. Clearly, $\left|\eta_{n, i}\right| \leq 1$. Now, we have:

(E1) for each $R>0, U_{n}(\xi) \neq 0$ in $\Delta(0, R)$ for sufficiently large $n$;

(E2) $U_{n}(1)=\infty$;

(E3) $U_{n}^{(k)}(\xi) \neq a_{n}^{m} s_{n}^{m} h_{n}\left(a_{n}^{m} s_{n}^{m} \xi\right)$ and $a_{n}^{m} s_{n}^{m} h_{n}\left(a_{n}^{m} s_{n}^{m} \xi\right) \stackrel{\chi}{\Rightarrow} \frac{1}{\xi^{m}}$ in $\mathbb{C}$;

(E4) $U_{n}(\xi)$ has only $m-k$ poles $\eta_{n, i}$ on $\bar{\Delta}$, where $i=1,2, \ldots, m-k$.

In fact, (E1) holds by (5.5). By Lemma 3.3, (E1), and (E3), we find that $U_{n}(\xi)$ is normal in $\mathbb{C}$. We assume that $U_{n}(\xi) \stackrel{x}{\Rightarrow} U(\xi)$ in $\mathbb{C}$. Obviously, $U(1)=\infty$ by (E2).

Subcase 1.1.2.1. $U(\xi)$ is a meromorphic function in $\mathbb{C}$.

By Lemma 3.1, we have either $U^{(k)}(\zeta) \equiv \frac{1}{\xi^{m}}$ or $U^{(k)}(\xi) \neq \frac{1}{\xi^{m}}$ in $\mathbb{C}$. Since $U(1)=\infty$, $U^{(k)}(\xi) \neq \frac{1}{\xi^{m}}$ in $\mathbb{C}$. By Lemma 2.8 and Lemma $2.9, U(\xi)$ is a constant function. This contradicts $U(1)=\infty$.

Subcase 1.1.2.2. $U(\xi) \equiv \infty$ in $\mathbb{C}$.

Set $U_{n}^{*}(\xi):=U_{n}(\xi) \cdot \prod_{i=1}^{m-k}\left(\xi-\eta_{n, i}\right)$. By the maximum principle applied to $\frac{1}{U_{n}^{*}(\xi)}$, we get that

$$
U_{n}^{*}(\xi) \stackrel{\chi}{\Rightarrow} \infty \quad \text { in } \mathbb{C} .
$$

Set $F_{n}^{*}(\zeta):=F_{n}(\zeta) \cdot \prod_{i=1}^{m-k}\left(\zeta-\gamma_{n, i}\right)=F_{n}(\zeta) \cdot \prod_{i=1}^{m-k}\left(\zeta-s_{n} \eta_{n, i}\right)$. By (5.5), $F_{n}^{*}(\zeta)$ has no poles in $\Delta\left(0, \frac{1}{2}\right)$ for sufficiently large $n$. By the maximum principle,

$$
F_{n}^{*}(\zeta) \stackrel{\chi}{\Rightarrow} \quad \frac{1+P_{k-1}(\zeta) \zeta^{m-k}}{(-m+k)(-m+k-1) \cdots(-m+1)} \quad \text { in } \Delta\left(0, \frac{1}{2}\right)
$$

Hence,

$$
F_{n}^{*}(0) \rightarrow \frac{1}{(-m+k)(-m+k-1) \cdots(-m+1)} \quad \text { as } n \rightarrow \infty .
$$

On the other hand,

$$
\begin{aligned}
F_{n}^{*}\left(s_{n} \xi\right) & =F_{n}\left(s_{n} \xi\right) \cdot \prod_{i=1}^{m-k}\left(s_{n} \xi-\gamma_{n, i}\right)=F_{n}\left(s_{n} \xi\right) \cdot \prod_{i=1}^{m-k}\left(s_{n} \xi-s_{n} \eta_{n, i}\right) \\
& =s_{n}^{m-k} F_{n}\left(s_{n} \xi\right) \cdot \prod_{i=1}^{m-k}\left(\xi-\eta_{n, i}\right)=U_{n}^{*}(\xi) \stackrel{\chi}{\Rightarrow} \infty
\end{aligned}
$$

in $\mathbb{C}$, and hence $F_{n}^{*}(0) \rightarrow \infty$, which contradicts (5.7).

Subcase 1.2. There exists a subsequence of $\left\{f_{n}(z)\right\}$ (still denoted by $\left.\left\{f_{n}(z)\right\}\right)$ such that $f_{n}(0)=0$ for each $n$. 
Do as in Subcase 1.1.1, we may assume that $y_{n}(\neq 0)$ is the pole of $f_{n}(z)$ of smallest modulus and $y_{n} \rightarrow 0$. Set $G_{n}(\zeta):=y_{n}^{m-k} f_{n}\left(y_{n} \zeta\right)$. We have:

(F1) $G_{n}(\zeta)$ is holomorphic function in $\Delta$;

(F2) $G_{n}(1)=\infty$;

(F3) all zeros of $G_{n}(\zeta)$ have multiplicity at least $k+1$;

(F4) $G_{n}^{(k)}(\zeta) \neq y_{n}^{m} h_{n}\left(y_{n} \zeta\right)$ and $y_{n}^{m} h_{n}\left(y_{n} \zeta\right) \stackrel{x}{\Rightarrow} \frac{1}{\zeta^{m}}$ in $\mathbb{C}$.

By Lemma 3.8 and Lemma 3.4, $\left\{G_{n}(\zeta)\right\}$ is normal in $\Delta$ and quasinormal in $\mathbb{C}$. Thus, there exist a subsequence of $\left\{G_{n}(\zeta)\right\}$ (still denoted by $\left.\left\{G_{n}(\zeta)\right\}\right)$ and $D_{3} \subset \mathbb{C}$ such that:

(G1) $D_{3}$ has no accumulation points in $\mathbb{C}$;

(G2) for each $\zeta_{0} \in D_{3}$, no subsequence of $\left\{G_{n}(\zeta)\right\}$ is normal at $\zeta_{0}$;

(G3) $G_{n}(\zeta) \stackrel{\chi}{\Rightarrow} G(\zeta)$ in $\mathbb{C} \backslash D_{3}$.

Obviously, $D_{3} \cap \Delta=\emptyset$ and all zeros of $G(\zeta)$ have multiplicity at least $k+1$ in $\mathbb{C} \backslash D_{3}$.

Obviously, $G(0)=\lim _{n \rightarrow \infty} G_{n}(0)=y_{n}^{m-k} f_{n}(0)=0$, so $G(z)$ is a meromorphic function in $\mathbb{C} \backslash D_{3}$. By Lemma 3.1 and (F4), either $G^{(k)}(\zeta) \equiv \frac{1}{\zeta^{m}}$ or $G^{(k)}(\zeta) \neq \frac{1}{\zeta^{m}}$ in $\mathbb{C} \backslash D_{3}$.

Subcase 1.2.1. $D_{3}$ is an empty set.

By (F2), $G(1)=\infty$. If $G^{(k)}(\zeta) \neq \frac{1}{\zeta^{m}}$, then by Theorem 2.8 and Lemma 2.9, $G(\zeta)$ is a constant function, which contradicts that $G(1)=\infty$. If $G^{(k)}(\zeta)-\frac{1}{\zeta^{m}} \equiv 0$, then we have $G(0)=\infty$, which contradicts $G(0)=0$.

Subcase 1.2.2. $D_{3}$ is not an empty set.

Let $\zeta_{0} \in E$. Since $D_{3} \cap \Delta=\emptyset$, by Lemma 2.5 , we have $G^{(k)}(\zeta)=\frac{1}{\zeta^{m}}$, which contradicts $G(0)=0$.

Case 2. $h(0)=0$.

In this case, we will show that $(* \mathrm{f})$ and $(* \mathrm{~g})$ hold. Clearly, we have $h(z) \neq 0, \infty$ in $\Delta^{\prime}$, $h_{n}(z) \neq 0, \infty$ in $\Delta^{\prime}$, and $h(0)=h_{n}(0)=0$.

We claim that for each $\delta>0$, there exists at least one zero of $f_{n}$ in $\Delta^{\prime}(0, \delta)$ for sufficiently large $n$. Otherwise, there exist $\delta^{*}(>0)$ and a subsequence of $\left\{f_{n}\right\}$ (still denoted by $\left\{f_{n}\right\}$ ) such that $f_{n}(z) \neq 0$ in $\Delta^{\prime}\left(0, \delta^{*}\right)$. Since $f_{n}^{(k)}(z) \neq h_{n}(z)$ and all the zeros of $\left\{f_{n}\right\}$ have multiplicity at least $k+1$, we have $f_{n}(0) \neq 0$, and hence $f_{n}(z) \neq 0$ in $\Delta\left(0, \delta^{*}\right)$. By Lemma 3.3, $\left\{f_{n}\right\}$ is normal at 0 , which contradicts $(* c)$.

Taking a subsequence and renumbering, we may assume that $a_{n}(\neq 0)$ is the zero of $f_{n}$ of smallest modulus and $a_{n} \rightarrow 0$. Set $F_{n}(\zeta):=\frac{f_{n}\left(a_{n} \zeta\right)}{a_{n}^{k+l}}$. We have:

(a1) $F_{n}(\zeta) \neq 0$ in $\Delta$;

(a2) all zeros of $F_{n}(\zeta)$ have multiplicity at least $k+1$ and $F_{n}(1)=0$;

(a3) $F_{n}^{(k)}(\zeta) \neq \zeta^{l} \hat{h}\left(a_{n} \zeta\right)$ and $\zeta^{l} \hat{h}\left(a_{n} \zeta\right) \stackrel{\gamma}{\Rightarrow} \zeta^{l}$ in $\mathbb{C}$.

By Lemma 3.3 and Lemma 3.4, $\left\{F_{n}(\zeta)\right\}$ is normal in $\Delta$ and quasinormal in $\mathbb{C}$. Thus, there exist a subsequence of $\left\{F_{n}(\zeta)\right\}$ (still denoted by $\left\{F_{n}(\zeta)\right\}$ ) and $D_{4} \subset \mathbb{C}$ such that:

(b1) $D_{4}$ has no accumulation points in $\mathbb{C}$;

(b2) for each $\zeta_{0} \in D_{4}$, no subsequence of $\left\{F_{n}(\zeta)\right\}$ is normal at $\zeta_{0}$;

(b3) $F_{n}(\zeta) \stackrel{x}{\Rightarrow} F(\zeta)$ in $\mathbb{C} \backslash D_{4}$.

Obviously, $D_{4} \cap \Delta=\emptyset$ and all zeros of $F(\zeta)$ have multiplicity at least $k+1$ in $\mathbb{C} \backslash D_{4}$.

Subcase 2.1. $1 \notin D_{4}$.

By (a2), $F(1)=F^{(k)}(1)=0$, and hence $F(\zeta)$ is a meromorphic function in $\mathbb{C} \backslash D_{4}$.

We claim that $D_{4}=\emptyset$. Otherwise, let $\zeta_{0} \in D_{4}$. Since $D_{4} \cap \Delta=\emptyset$, by Lemma $2.5, F^{(k)}(\zeta)=$ $\zeta^{k}$, which contradicts that $F^{(k)}(1)=0$. 
By Lemma 3.1 and (a3), either $F^{(k)}(\zeta) \equiv \zeta^{k}$ or $F^{(k)}(\zeta) \neq \zeta^{k}$ in $\mathbb{C}$. Since $F^{(k)}(1)=0$, we have $F^{(k)}(\zeta) \neq \zeta^{k}$ in $\mathbb{C}$. By Lemma 2.8 and Lemma 3.5, we have

$$
F(\zeta)=\frac{\prod_{i=1}^{t+1+l}\left(\zeta-\alpha_{i}\right)}{(l+k)(l+k-1) \cdots(l+1)(\zeta-\beta)^{t+1-k}}
$$

where $t \geq k$ is an integer, $\beta \in \mathbb{C}$, and $\alpha_{i} \neq 0, \beta(1 \leq i \leq t+1+l)$. Thus, we have

$$
F_{n}(\zeta) \stackrel{\chi}{\Rightarrow} \quad \frac{\prod_{i=1}^{t+1+l}\left(\zeta-\alpha_{i}\right)}{(l+k)(l+k-1) \cdots(l+1)(\zeta-\beta)^{t+1-k}} \quad \text { in } \mathbb{C} .
$$

By Hurwitz's theorem, there exist sequences $\zeta_{n, i} \rightarrow \alpha_{i}$ and $\eta_{n, j} \rightarrow \beta$ (counting multiplicities of zeros and poles, respectively) such that for sufficiently large $n, F_{n}\left(\zeta_{n, i}\right)=0$ and $F_{n}\left(\eta_{n, j}\right)=\infty$, where $i=1,2, \ldots, t+1+l$ and $j=1,2, \ldots, t+1-k$. set $z_{n, i}:=a_{n} \zeta_{n, i}$. Thus, $f_{n}\left(z_{n, i}\right)=0$ and $z_{n, i} \rightarrow 0$, where $i=1,2, \ldots, t+1+l$. Set

$$
B_{n}:=\left\{z_{n, 1}, z_{n, 2}, \ldots, z_{n, t+1+l}\right\} \quad \text { (where the same elements are admissible). }
$$

Subcase 2.1.1. For each $\delta>0, f_{n}$ has at least $t+2+l$ zeros (counting multiplicities) in $\Delta(0, \delta)$ for sufficiently large $n$.

Taking a subsequence and renumbering, we may assume that $b_{n}(\neq 0)$ is the zero of $f_{n}$ of smallest modulus in $\Delta \backslash B_{n}$ and $b_{n} \rightarrow 0$. Clearly, $F_{n}\left(\frac{b_{n}}{a_{n}}\right)=0$ and $\frac{b_{n}}{a_{n}} \neq \zeta_{n, i}$ for $i=1,2, \ldots, t+$ $1+l$. By Hurwitz's theorem and (5.8), $\frac{a_{n}}{b_{n}} \rightarrow 0$ as $n \rightarrow \infty$. Set $G_{n}(\zeta):=\frac{f_{n}\left(b_{n} \zeta\right)}{b_{n}^{k+l}}$. We have, for sufficiently large $n$ :

(c1) $G_{n}(\zeta)$ has only $t+1+l$ zeros $\frac{a_{n} \zeta_{n, i}}{b_{n}}$ in $\Delta$. Obviously, $\left|\frac{a_{n} \zeta_{n, i}}{b_{n}}\right| \rightarrow 0$;

(c2) all zeros of $G_{n}(\zeta)$ have multiplicity at least $k+1$ and $G_{n}(1)=0$;

(c3) $G_{n}^{(k)}(\zeta) \neq \zeta^{l} \hat{h}\left(b_{n} \zeta\right)$ and $\zeta^{l} \hat{h}\left(b_{n} \zeta\right) \stackrel{\chi}{\Rightarrow} \zeta^{l}$ in $\mathbb{C}$.

By Lemma 3.3 and Lemma 3.4, $\left\{G_{n}(\zeta)\right\}$ is normal in $\Delta^{\prime}$ and quasinormal in $\mathbb{C}$. Thus, there exist a subsequence of $\left\{G_{n}(\zeta)\right\}$ (still denoted by $\left.\left\{G_{n}(\zeta)\right\}\right)$ and $D_{5} \subset \mathbb{C}$ such that:

(d1) $D_{5}$ has no accumulation points in $\mathbb{C}$;

(d2) for each $\zeta_{0} \in D_{5}$, no subsequence of $\left\{G_{n}(\zeta)\right\}$ is normal at $\zeta_{0}$;

(d3) $G_{n}(\zeta) \stackrel{x}{\Rightarrow} G(\zeta)$ in $\mathbb{C} \backslash D_{5}$.

Obviously, $D_{5} \cap \Delta^{\prime}=\emptyset$ and all zeros of $G(\zeta)$ have multiplicity at least $k+1$ in $\mathbb{C} \backslash D_{5}$.

Set

$$
G_{n}^{*}(\zeta):=G_{n}(\zeta) \frac{\prod_{j=1}^{t+1-k}\left(\zeta-\frac{a_{n} \eta_{n, j}}{b_{n}}\right)}{\prod_{i=1}^{t+1+l}\left(\zeta-\frac{a_{n} \zeta_{n, i}}{b_{n}}\right)} \quad \text { and } \quad F_{n}^{*}(\zeta)=F_{n}(\zeta) \frac{\prod_{j=1}^{t+1-k}\left(\zeta-\eta_{n, j}\right)}{\prod_{i=1}^{t+1+l}\left(\zeta-\zeta_{n, i}\right)}
$$

By $(5.8), G_{n}^{*}\left(\frac{a_{n} \zeta}{b_{n}}\right)=F_{n}^{*}(\zeta) \Rightarrow \frac{1}{(l+k)(l+k-1) \cdots(l+1)}$ in $\mathbb{C}$. Hence

$$
G_{n}^{*}(0) \rightarrow \frac{1}{(l+k)(l+k-1) \cdots(l+1)}
$$

Subcase 2.1.1.1. $G(\zeta) \equiv \infty$ in $\mathbb{C} \backslash D_{5}$.

Obviously, $G_{n}^{*}(\zeta)$ has no zeros in $\Delta$ for sufficiently large $n$. Applying the maximum principle to $\frac{1}{G_{n}^{*}(\zeta)}$, we see that $G_{n}^{*}(\zeta) \stackrel{x}{\Rightarrow} \infty$ in $\Delta$, which contradicts (5.9).

Subcase 2.1.1.2. $G(\zeta)$ is a meromorphic function in $\mathbb{C} \backslash D_{5}$. 
We claim that

$$
G(\zeta)=\frac{\zeta^{l+k}}{(l+k)(l+k-1) \cdots(l+1)} \quad \text { in } \mathbb{C} \backslash D_{5} .
$$

By Lemma 3.2, $G^{(k)}(\zeta) \equiv \zeta^{l}$ in $\Delta^{\prime}$, and then $G(\zeta)=\frac{\zeta^{l+k}+P_{k-1}(\zeta)}{(l+k)(l+k-1) \cdots(l+1)}$ in $\Delta^{\prime}$, where $P_{k-1}(z)$ is a polynomial of degree $\leq k-1$. Since $G_{n}^{*}(\zeta)$ has no zeros in $\Delta$ for sufficiently large $n$, applying the maximum principle to $\frac{1}{G_{n}^{*}(\zeta)}, G_{n}^{*}(\zeta) \Rightarrow G^{*}(\zeta)=\frac{\zeta^{l+k}+P_{k-1}(\zeta)}{(l+k)(l+k+1) \cdots(l+1)} \frac{1}{\zeta^{l+k}}$ in $\Delta$. By $(5.9)$,

$$
G^{*}(0)=\left.\frac{\zeta^{l+k}+P_{k-1}(\zeta)}{(l+k)(l+k-1) \cdots(l+1)} \frac{1}{\zeta^{l+k}}\right|_{\zeta=0}=\frac{1}{(l+k)(l+k-1) \cdots(l+1)} .
$$

Since $P_{k-1}(z)$ is a polynomial of degree $\leq k-1$, we have $P_{k-1}(\zeta) \equiv 0$ by (5.11). Thus our claim is proved.

Suppose that $1 \notin D_{5}$. By (c2), we have $G(1)=0$, which contradicts (5.10). Suppose that $1 \in D_{5}$. By Lemma 2.5, $G^{(k-1)}(\zeta)=\int_{1}^{\zeta} \xi^{l} \mathrm{~d} \xi=\frac{\zeta^{l+1}-1}{l+1}$. However, we have $G^{(k-1)}(\zeta)=\frac{\zeta^{l+1}}{l+1}$ by (5.10). This is a contradiction.

Subcase 2.1.2. There exists $\delta>0$ such that $f_{n}$ has exactly $t+1+l$ zeros (counting multiplicities) in $\Delta(0, \delta)$ for sufficiently large $n$.

Taking a subsequence and renumbering, we may assume that $f_{n}$ has exactly $t+1+l$ zeros (counting multiplicities) in $\Delta(0, \delta)$ for all $n$. Now, $(* \mathrm{f})$ holds with $r_{\tilde{a}}=\delta$ and $N_{\tilde{a}}=m+k+2$. Next, we will show that $(* \mathrm{~g})$ also holds.

Set

$$
f_{n}^{*}(z):=f_{n}(z) \frac{\prod_{j=1}^{t+1-k}\left(z-a_{n} \eta_{n, j}\right)}{\prod_{i=1}^{t+1+l}\left(z-a_{n} \zeta_{n, i}\right)} \quad \text { and } \quad F_{n}^{*}(\zeta)=F_{n}(\zeta) \frac{\prod_{j=1}^{t+1-k}\left(\zeta-\eta_{n, j}\right)}{\prod_{i=1}^{t+1+l}\left(\zeta-\zeta_{n, i}\right)}
$$

Clearly, $f_{n}^{*}(z)$ has no zeros in $\Delta(0, \delta)$. By $(5.8), f_{n}^{*}\left(a_{n} \zeta\right)=F_{n}^{*}(\zeta) \Rightarrow \frac{1}{(l+k)(l+k-1) \cdots(l+1)}$ in $\mathbb{C}$, and hence

$$
f_{n}^{*}(0) \rightarrow \frac{1}{(l+k)(l+k-1) \cdots(l+1)} .
$$

Subcase 2.1.2.1 $f(z) \equiv \infty$ in $D \backslash E$.

By the maximum principle applied to $\frac{1}{f_{n}^{*}(z)}$, we have $f_{n}^{*}(z) \stackrel{x}{\Rightarrow} \infty$ in $\Delta(0, \delta)$, which contradicts (5.12).

Subcase 2.1.2.2. $f(z)$ is a meromorphic function in $D \backslash E$.

By Lemma 3.2, $f^{(k)}(z)=h(z)$ in $\Delta^{\prime}(0, \delta)$. By the maximum principle applied to $\frac{1}{f_{n}^{*}(z)}$, $f_{n}^{*}(z) \stackrel{\chi}{\Rightarrow} \frac{f(z)}{z^{l+k}}$ in $\Delta(0, \delta)$. Hence by $(5.12)$,

$$
\left.f_{n}^{*}(0) \rightarrow \frac{f(z)}{z^{l+k}}\right|_{\zeta=0}=\frac{1}{(l+k)(l+k-1) \cdots(l+1)} .
$$

Equation (5.13) implies that $z=0$ is a zero of order $l+k$ of $f(z)$. Thus we have

$$
f(z)=\int_{0}^{z} \int_{0}^{\zeta_{1}} \cdots \int_{0}^{\zeta_{k-1}} h\left(\zeta_{k}\right) \mathrm{d} \zeta_{k} \mathrm{~d} \zeta_{k-1} \cdots \mathrm{d} \zeta_{1}
$$

Subcase 2.2. $1 \in D_{4}$. 
By Lemma 2.5,

$$
\begin{aligned}
& F^{(k-1)}(\zeta)=\int_{1}^{\zeta} \xi^{l} \mathrm{~d} \xi=\frac{\zeta^{l+1}-1}{l+1} \\
& F^{(k-2)}(\zeta)=\int_{1}^{\zeta} \frac{\xi^{l+1}-1}{l+1} \mathrm{~d} \xi=\frac{\zeta^{l+2}}{(l+2)(l+1)}-\frac{\zeta}{l+1}+\frac{1}{(l+2)}
\end{aligned}
$$

We claim that $D_{4}=\{1\}$. Otherwise, there exists $\zeta_{0} \in E_{1}$ and $\zeta_{0} \neq 0,1$. By Lemma 2.5, in $\mathbb{C} \backslash D_{4}$

$$
F^{(k-1)}(\zeta)=\int_{\zeta 0}^{\zeta} \xi^{l} \mathrm{~d} \xi=\frac{\zeta^{l+1}-\zeta_{0}^{l+1}}{l+1}
$$

By (5.14) and (5.16), we obtain $\zeta_{0}{ }^{l+1}=1$. Furthermore, by Lemma 2.5, in $\mathbb{C} \backslash D_{4}$,

$$
F^{(k-2)}(\zeta)=\int_{\zeta 0}^{\zeta} \frac{\xi^{l+1}-1}{l+1} \mathrm{~d} \xi=\frac{\zeta^{l+2}}{(l+2)(l+1)}-\frac{\zeta}{l+1}+\frac{\zeta_{0}}{(l+2)}
$$

By (5.15) and (5.17), we get $\zeta_{0}=1$. A contradiction. Thus $D_{4}=\{1\}$.

By Lemma 2.5, $F(z)$ can extend to an analytic function in $\mathbb{C}$ and thus is a polynomial of degree $l+k$. Since 1 is a zero of order $k$ of $F, F$ must have at least one zero which is distinct from 1 . Let $e_{1}(\neq 1)$ is a zero of $F(z)$. Since all zeros of $F(\zeta)$ have multiplicity at least $k+1$ in $\mathbb{C} \backslash\{1\}$, we have $F^{(k-1)}\left(e_{1}\right)=0$ and $F^{(k)}\left(e_{1}\right)=0$. Then $e_{1}$ is a multiple zero of $F^{(k-1)}(z)$. However, by (5.14), $F^{(k-1)}(z)$ only has zeros of order 1 . This is a contradiction.

Case $3 . h(0) \neq 0, \infty$.

Obviously, $(* g)$ holds by Lemma 2.5 . Next, we will show that $(* \mathrm{f})$ also holds.

Since $\left\{f_{n}\right\}$ is not normal at 0 , it follows from Lemma 2.2 that we can extract a subsequence (still denoted by $\left\{f_{n}\right\}$ ), points $z_{n} \rightarrow 0$, and positive numbers $\rho_{n} \rightarrow 0$ such that

$$
g_{n}(\zeta)=\rho_{n}^{-k} f_{n}\left(z_{n}+\rho_{n} \zeta\right) \stackrel{\chi}{\Rightarrow} g(\zeta) \quad \text { in } \mathbb{C}
$$

where $g$ is a nonconstant meromorphic function of finite order in $\mathbb{C}$, all of whose zeros have multiplicity at least $k+1$. Since $g_{n}^{(k)}(\zeta)=f_{n}^{(k)}\left(z_{n}+\rho_{n} \zeta\right) \neq h_{n}\left(z_{n}+\rho_{n} \zeta\right) \Rightarrow h(0)$ and $g_{n}^{(k)} \Rightarrow g^{(k)}(\zeta)$ on the complement of the poles of $g(\zeta)$, either $g^{(k)}(\zeta) \neq h(0)$ or $g^{(k)}(\zeta) \equiv h(0)$ in $\mathbb{C}$ by Hurwitz's theorem. The latter case is not possible, as this would contradict the fact that all zeros of $g$ have multiplicity at least $k+1$. Thus $g^{(k)}(\zeta) \neq h(0)$ in $\mathbb{C}$. By Lemma 2.11,

$$
g(\zeta)=\frac{h(0)}{k !} \frac{(\zeta-a)^{k+1}}{\zeta-b}
$$

for distinct complex numbers $a$ and $b$. It now follows from the argument principle that there exists a sequence $\xi_{n} \rightarrow a$ such that, for sufficiently large $n, g_{n}\left(\xi_{n}\right)=0$. Thus, writing $z_{n, 0}=z_{n}+\rho_{n} \xi_{n}$, we have $z_{n, 0} \rightarrow 0$. By (5.18) and (5.19), the multiplicity of $z_{n, 0}$ as a zero of $f_{n}$ is exactly $k+1$ for sufficiently large $n$. By Lemma 2.5 , there exists $r_{1}>0$ such that $f_{n}$ has a single (multiple) zero in $\Delta\left(0, r_{1}\right)$ for sufficiently large $n$. Thus for sufficiently large $n, f_{n}$ has a single zero $z_{n, 0}$ of order exactly $k+1$ in $\Delta\left(0, r_{1}\right)$. Now, $(* \mathrm{f})$ holds with $r_{\tilde{a}}=r_{1}$ and $N_{\tilde{a}}=k+2$. 


\section{Proof of Proposition 1.2}

Proof By Lemma 3.4, $\left\{f_{n}\right\}$ is quasinormal in $D$. Hence for each subsequence $\left\{f_{n_{k}}\right\}$ of $\left\{f_{n}\right\}$, there exists a subsequence of $\left\{f_{n_{k}}\right\}$ (still denoted by $\left\{f_{n_{k}}\right\}$ ) and a corresponding point set $E$ which has no accumulation points in $D$ such that:

(a) $f_{n_{k}}(z) \stackrel{\chi}{\Rightarrow} f(z)$ in $D \backslash E$, where $f(z)$ is meromorphic or identically infinite there;

(b) for each $\tilde{a} \in E$, no subsequence of $\left\{f_{n_{k}}\right\}$ is normal at $\tilde{a}$.

By Lemma 3.9, we have:

(c) for each $\tilde{a} \in E, h(\tilde{a}) \neq \infty$;

(d) for each $\tilde{a} \in E$, there exist $r_{\tilde{a}}>0$ and $N_{\tilde{a}}>0$ such that for sufficiently large $k$, $n\left(\Delta\left(\tilde{a}, r_{\tilde{a}}\right), \frac{1}{f_{n_{k}}}\right)<N_{\tilde{a}}$, where $r_{\tilde{a}}$ and $N_{\tilde{a}}$ only depend on $\tilde{a}$;

(e) for each $\tilde{a} \in E, f(z)=\int_{\tilde{a}}^{z} \int_{\tilde{a}}^{\zeta_{1}} \cdots \int_{\tilde{a}}^{\zeta_{k-1}} H^{\prime}\left(\zeta_{k}\right) \mathrm{d} \zeta_{k} \mathrm{~d} \zeta_{k-1} \cdots \mathrm{d} \zeta_{1}$ in $D \backslash E$.

Clearly, we see that (1), (2), (3), and (4) hold.

Suppose that $|E|>v$ and $z_{1}, z_{2}, \ldots, z_{v+1}$ are $v+1$ distinct points in $E$. Since for each $a^{*} \in \mathbb{C}$, $\bar{n}\left(D, \frac{1}{H(z)-a^{*}}\right) \leq v$, there exist $i, j \in \mathbb{N}$ such that $1 \leq i<j \leq v+1$ and $H\left(z_{i}\right) \neq H\left(z_{j}\right)$. However, $f^{(k-1)}(z)=H(z)-H\left(z_{i}\right)$ and $f^{(k-1)}(z)=H(z)-H\left(z_{j}\right)$ by $(\mathrm{e})$. Thus $H\left(z_{i}\right)=H\left(z_{j}\right)$. A contradiction. This completes the proof of Proposition 1.2.

\section{Proof of Theorem 1.3}

Proof We argue by contradiction. Suppose that the equation $f^{(k)}(z)=\alpha(z)$ has at most finitely many solutions. Let $R(z) \sim c_{0} z^{d}$ as $z \rightarrow \infty$, where $c_{0} \in \mathbb{C} \backslash\{0\}$ and $d \in \mathbb{Z}$.

Clearly, $T(r, R)=O(\log r)$ and $T\left(r, \frac{1}{R}\right)=O(\log r)$ as $r \rightarrow \infty$. By Lemma 2.1, $T(r, h)=$ $A r^{2}(1+o(1))$ as $r \rightarrow \infty$, where $A>0$ is a constant. By standard results in Nevanlinna theory, $T(r, h)=T\left(r, \frac{\alpha}{R}\right) \leq T(r, \alpha)+T\left(r, \frac{1}{R}\right)$ and $T(r, \alpha) \leq T(r, R)+T(r, h)$ as $r \rightarrow \infty$. Thus, $T(r, \alpha)=A r^{2}(1+o(1))$ as $r \rightarrow \infty$. Since $T(r, \alpha)=o\{T(r, f)\}$ as $r \rightarrow \infty$, we have $\overline{\lim }_{r \rightarrow \infty} \frac{T(r, f)}{r^{2}}=\infty$.

Set $g(z):=\frac{f(z)}{z^{d}}$. By Lemma 3.6, there exist points $t_{n} \rightarrow \infty$ and positive numbers $\varepsilon_{n} \rightarrow 0$ such that

$$
S\left(\Delta\left(t_{n}, \varepsilon_{n}\right), g\right) \rightarrow \infty \quad \text { as } n \rightarrow \infty
$$

and

$$
\frac{f\left(t_{n}\right)}{t_{n}^{d}} \rightarrow 0 \quad \text { and } \quad \frac{f^{(k)}\left(t_{n}\right)}{t_{n}^{d}} \rightarrow \infty \quad \text { as } n \rightarrow \infty
$$

Let $\omega_{1}, \omega_{2}$ be the two fundamental periods of $h(z)$ and $P(0 \in P)$ be a fundamental parallelogram of $h(z)$. There exist integers $i_{n}$ and $j_{n}$ such that $z_{n} \in P$, where $z_{n}=t_{n}-i_{n} \omega_{1}-j_{n} \omega_{2}$. There exists a subsequence of $\left\{z_{n}\right\}$ (still denoted by $\left\{z_{n}\right\}$ ) such that $z_{n} \rightarrow z_{0}$ as $n \rightarrow \infty$. Set

$$
\begin{aligned}
g_{n}(z) & :=g\left(z+i_{n} \omega_{1}+j_{n} \omega_{2}\right), \\
f_{n}(z) & :=\frac{f\left(z+i_{n} \omega_{1}+j_{n} \omega_{2}\right)}{t_{n}^{d}} .
\end{aligned}
$$

Clearly, we have $S\left(\Delta\left(z_{n}, \varepsilon_{n}\right), g_{n}\right)=S\left(\Delta\left(t_{n}, \varepsilon_{n}\right), g\right), f_{n}\left(z_{n}\right)=\frac{f\left(t_{n}\right)}{t_{n}^{d}}$, and $f_{n}^{(k)}\left(z_{n}\right)=\frac{f^{(k)}\left(t_{n}\right)}{t_{n}^{d}}$. By $(7.1)$ and (7.2), we have

$$
S\left(\Delta\left(z_{n}, \varepsilon_{n}\right), g_{n}\right) \rightarrow \infty \quad \text { as } n \rightarrow \infty,
$$




$$
f_{n}\left(z_{n}\right) \rightarrow 0 \quad \text { and } \quad f_{n}^{(k)}\left(z_{n}\right) \rightarrow \infty \quad \text { as } n \rightarrow \infty
$$

There exists $R>0$ such that $\bar{P} \subset \Delta(0, R)$ and $\Delta\left(z_{n}, \varepsilon_{n}\right) \subset \Delta(0, R)$ for each $n$. Set $D:=$ $\Delta(0, R)$. Obviously, we have $z_{0} \in D$. By assumption, for sufficiently large $n$,

$$
f_{n}^{(k)}(z)=\frac{f^{(k)}\left(z+i_{n} \omega_{1}+j_{n} \omega_{2}\right)}{t_{n}^{d}} \neq \frac{R\left(z+i_{n} \omega_{1}+j_{n} \omega_{2}\right) h(z)}{t_{n}^{d}}, \quad z \in D .
$$

For each $z \in D$,

$$
\left|t_{n}-\left(z+i_{n} \omega_{1}+j_{n} \omega_{2}\right)\right|=\left|\left(z_{n}+i_{n} \omega_{1}+j_{n} \omega_{2}\right)-\left(z+i_{n} \omega_{1}+j_{n} \omega_{2}\right)\right|=\left|z_{n}-z\right|<2 R .
$$

So we have

$$
\frac{R\left(z+i_{n} \omega_{1}+j_{n} \omega_{2}\right)}{t_{n}^{d}} \rightarrow c_{0} \quad \text { as } n \rightarrow \infty
$$

Set

$$
T_{n}(z):=\frac{R\left(z+i_{n} \omega_{1}+j_{n} \omega_{2}\right) h(z)}{t_{n}^{d}} .
$$

Obviously, $T_{n}(z) \stackrel{\chi}{\Rightarrow} c_{0} h(z)$ in $D$, and for sufficiently large $n, c_{0} h$ and $T_{n}$ have the same zeros and poles with the same multiplicity in $D$.

Clearly, $\left\{f_{n}\right\}$ is a family of meromorphic functions in $D$ such that for sufficiently large $n$ :

(a1) all zeros of $\left\{f_{n}\right\}$ have multiplicity at least $k+1$ in $D$;

(a2) $T_{n}(z) \stackrel{\chi}{\Rightarrow} c_{0} h(z)$ in $D$, where $c_{0} h(z) \not \equiv 0, \infty$ in $D$;

(a3) $f_{n}^{(k)}(z) \neq T_{n}(z)$ in $D$.

It follows from Lemma 3.4 that $\left\{f_{n}\right\}$ is quasinormal in $D$. Hence there exists $\tau>0$ such that $\Delta\left(z_{0}, \tau\right) \subset D$ and $\left\{f_{n}\right\}$ is normal in $\Delta^{\prime}\left(z_{0}, \tau\right)$. Then there exists a subsequence of $\left\{f_{n}\right\}$ (still denoted by $\left.\left\{f_{n}\right\}\right)$ such that:

(b1) $c_{0} h(z)$ and $T_{n}(z)$ have the same zeros and poles with the same multiplicity in $\Delta\left(z_{0}, \tau\right)$

(b2) for all $n \in \mathbb{N}, f_{n}^{(k)}(z) \neq T_{n}(z)$ in $\Delta\left(z_{0}, \tau\right)$;

(b3) no subsequence of $\left\{f_{n}\right\}$ is normal at $z_{0}$;

(b4) all zeros of $\left\{f_{n}\right\}$ have multiplicity at least $k+1$ in $\Delta\left(z_{0}, \tau\right)$, and $f_{n}(z) \stackrel{x}{\Rightarrow} f(z)$ in $\Delta^{\prime}\left(z_{0}, \tau\right)$.

By (7.6), (b3) holds. By Lemma 3.9, we have:

(c1) $h\left(z_{0}\right) \neq \infty$;

(c2) there exist $\tau^{*} \in(0, \tau)$ and $M^{*}>0$ such that $n\left(\Delta\left(z_{0}, \tau^{*}\right), \frac{1}{f_{n}}\right)<M^{*}$ for sufficiently large $n$;

(c3) $f(z)=\int_{z_{0}}^{z} \int_{z_{0}}^{\zeta_{1}} \cdots \int_{z_{0}}^{\zeta_{k-1}} c_{0} h\left(\zeta_{k}\right) \mathrm{d} \zeta_{k} \mathrm{~d} \zeta_{k-1} \cdots \mathrm{d} \zeta_{1}$ in $\Delta^{\prime}\left(z_{0}, \tau\right)$.

By Lemma 2.3, (c2) and (c3), there exists $M_{1}>0$ such that for sufficiently large $n$,

$$
S\left(\Delta\left(z_{0}, \frac{\tau^{*}}{2}\right), f_{n}\right)<M_{1}
$$

Next, we will derive a contradiction with (7.5). 
By (7.3) and (7.4), $g_{n}(z)=f_{n}(z)\left(1+\frac{z-z_{n}}{t_{n}}\right)^{-d}$. Then

$$
g_{n}^{\#}(z)=\frac{\left|\left(1+\frac{z-z_{n}}{t_{n}}\right)^{d} f_{n}^{\prime}(z)-\left(1+\frac{z-z_{n}}{t_{n}}\right)^{d}\left(\frac{d}{t_{n}+z-z_{n}}\right) f_{n}(z)\right|}{\left|\left(1+\frac{z-z_{n}}{t_{n}}\right)^{d}\right|^{2}+\left|f_{n}(z)\right|^{2}},
$$

So

$$
\left[g_{n}^{\#}(z)\right]^{2} \leq \frac{2\left|\left(1+\frac{z-z_{n}}{t_{n}}\right)^{d} f_{n}^{\prime}(z)\right|^{2}}{\left(\left|\left(1+\frac{z-z_{n}}{t_{n}}\right)^{d}\right|^{2}+\left|f_{n}(z)\right|^{2}\right)^{2}}+\frac{2\left|\left(1+\frac{z-z_{n}}{t_{n}}\right)^{d}\left(\frac{d}{t_{n}+z-z_{n}}\right) f_{n}(z)\right|^{2}}{\left(\left|\left(1+\frac{z-z_{n}}{t_{n}}\right)^{d}\right|^{2}+\left|f_{n}(z)\right|^{2}\right)^{2}} .
$$

Using the simple inequality

$$
\frac{C}{C^{2}+x^{2}} \leq 2 \max (C, 1 / C) \frac{1}{1+x^{2}}
$$

for $C>0$, we have

$$
\frac{2\left|\left(1+\frac{z-z_{n}}{t_{n}}\right)^{d} f_{n}^{\prime}(z)\right|^{2}}{\left(\left|\left(1+\frac{z-z_{n}}{t_{n}}\right)^{d}\right|^{2}+\left|f_{n}(z)\right|^{2}\right)^{2}} \leq 2 \max \left(\left|\left(1+\frac{z-z_{n}}{t_{n}}\right)\right|^{2 d}, \frac{1}{\left|\left(1+\frac{z-z_{n}}{t_{n}}\right)\right|^{2 d}}\right)\left[f_{n}^{\#}(z)\right]^{2} .
$$

The second term on the right of $(7.8)$ is

$$
\frac{1}{2}\left|\frac{d}{t_{n}+z-z_{n}}\right|^{2}\left(\frac{2\left|\left(1+\frac{z-z_{n}}{t_{n}}\right)^{d} f_{n}(z)\right|}{\left|\left(1+\frac{z-z_{n}}{t_{n}}\right)^{d}\right|^{2}+\left|f_{n}(z)\right|^{2}}\right)^{2} \leq \frac{1}{2}\left|\frac{d}{t_{n}+z-z_{n}}\right|^{2} .
$$

Putting (7.8), (7.9), and (7.10) together, we have for $z \in \Delta\left(z_{0}, \frac{\tau^{*}}{2}\right)$ and sufficiently large $n$,

$$
\left[g_{n}^{\#}(z)\right]^{2} \leq 4\left[f_{n}^{\#}(z)\right]^{2}+1
$$

It follows from (7.7) and (7.11) that

$$
S\left(\Delta\left(z_{0}, \frac{\tau^{*}}{2}\right), g_{n}\right) \leq 4 M_{1}+\left(\frac{\tau^{*}}{2}\right)^{2}:=M_{2}
$$

which contradicts (7.5). This completes the proof of Theorem 1.3.

\section{Competing interests}

The author declares that there is no conflict of interests regarding the publication of this article.

\section{Acknowledgements}

This work was supported by the National Natural Science Foundation of China (No. 11001081) and the Scientific Research Foundation of CUIT (No. KYTZ201403).

Received: 29 January 2014 Accepted: 15 September 2014 Published: 13 Oct 2014

\section{References}

1. Akhiezer, NI: Elements of the Theory of Elliptic Functions (Moscow, 1970). Translations of Mathematical Monographs, vol. 79. Am. Math. Soc., Rhode Island (1990). ISBN:0-8218-4532-2

2. Chuang, CT: Normal Families of Meromorphic Functions. World Scientific, Singapore (1993)

3. Nevo, S, Pang, XC, Zalcman, L: Quasinormality and meromorphic functions with multiple zeros. J. Anal. Math. 101, $1-23(2007)$

4. Hayman, WK: Picard values of meromorphic functions and their derivatives. Ann. Math. 70, 9-42 (1959) 
5. Bergweiler, W, Eremenko, A: On the singularities of the inverse to a meromorphic function of finite order. Rev. Mat. Iberoam. 11, 355-373 (1995)

6. Pang, XC, Nevo, S, Zalcman, L: Derivatives of meromorphic functions with multiple zeros and rational functions. Comput. Methods Funct. Theory 8, 483-491 (2008)

7. Bank, SB, Langley, JK: On the value distribution theory of elliptic functions. Monatshefte Math. 98, 1-20 (1984)

8. Pang, XC, Zalcman, L: Normal families and shared values. Bull. Lond. Math. Soc. 32, 325-331 (2000)

9. Nevo, S: On theorems of Yang and Schwick. Complex Var. Elliptic Equ. 46, 315-321 (2001)

10. Zalcman, L: Normal families: new perspectives. Bull. Am. Math. Soc. 35, 215-230 (1998)

11. Nevo, S: Applications of Zalcman's lemma to $Q_{m}$-normal families. Analysis 21, 289-325 (2001)

12. Yang, $P, N e v o, S$ : Derivatives of meromorphic functions with multiple zeros and elliptic functions. Acta Math. Sin. Engl. Ser. 29, 1257-1278 (2013)

13. Chang, JM, Zalcman, L: Meromorphic functions that share a set with their derivatives. J. Math. Anal. Appl. 338 , 1020-1028 (2008)

14. Yang, P, Liu, XJ: On the $k$ th derivatives of meromorphic functions and rational functions. J. East China Norm. Univ. Natur. Sci. Ed. 4, 8-17 (2014)

15. $\mathrm{Xu}, \mathrm{Y}$ : Picard values and derivatives of meromorphic functions. Kodai Math. J. 28, 99-105 (2005)

16. Xu, Y: Normal families and exceptional functions. J. Math. Anal. Appl. 329, 1343-1354 (2007)

17. Wang, YF, Fang, ML: Picard values and normal families of meromorphic functions with multiple zeros. Acta Math. Sin. $14,17-26(1998)$

10.1186/1029-242X-2014-389

Cite this article as: Yang: A quasinormal criterion of meromorphic functions and its application. Journal of Inequalities and Applications 2014, 2014:389

\section{Submit your manuscript to a SpringerOpen ${ }^{\circ}$ journal and benefit from:}

- Convenient online submission

- Rigorous peer review

- Immediate publication on acceptance

- Open access: articles freely available online

- High visibility within the field

- Retaining the copyright to your article 